\title{
QCD susceptibilities and nuclear matter saturation in a chiral theory: Inclusion of pion loops
}

\author{
G. Chanfray \\ IPN Lyon, Université de Lyon, Université Lyon 1, CNRS/IN2P3, UMR5822, F-69622 Villeurbanne Cedex, France \\ M. Ericson \\ IPN Lyon, Université de Lyon, Université Lyon 1, CNRS/IN2P3, UMR5822, F-69622 Villeurbanne Cedex, France and \\ Theory Division, CERN, CH-12111 Geneva, Switzerland \\ (Received 31 October 2006; published 25 January 2007)
}

\begin{abstract}
We derive the equation of state of symmetric nuclear matter in a relativistic theory with $\sigma$ and $\omega$ exchange. We take a chiral version of this model which insures all the chiral constraints. Going beyond the mean field approach we introduce the effects of the pion loops. For the parameters of the model, in order to fix those linked to pion exchange, we exploit the most recent information on the short-range part of the spin-isospin interaction. For those linked to the scalar meson exchange we make use of an analysis of lattice results on the nucleon mass evolution with the quark mass. With these inputs we are able reach a correct description of the saturation properties. From the equation of state of symmetric nuclear matter we also derive the density dependence of the quark condensate and of the QCD susceptibilities.
\end{abstract}

DOI: 10.1103/PhysRevC.75.015206

PACS number(s): 24.85.+p, 12.39.Fe, 13.75.Cs, 21.65.+f

\section{INTRODUCTION}

Relativistic theories of nuclear matter are needed for the prediction of the equation of state of nuclear matter at large densities, or of other quantities such as the QCD quark condensate or the QCD susceptibilities. They find a natural framework in the relativistic $\sigma$ and $\omega$ exchange model [1]. One important aspect is its chiral nature, especially if it also has to be used for the investigation of QCD related quantities such as the quark condensate. The approach that we follow in this work for symmetric nuclear matter is based on the chiral version of the $\sigma$ and $\omega$ model that we have proposed in Ref. [2]. Here the sigma model is formulated in a nonlinear version but with the presence of a chiral singlet scalar field $s$. The nuclear binding is provided by this chiral invariant field and in this way all chiral constraints are automatically satisfied. However it is known that in such chiral theories attractive tadpole diagrams destroy saturation $[3,4]$. Hence in order to achieve saturation we complement our model, as in our previous work [5], by the introduction of the scalar nucleonic response first introduced in the quark-meson coupling model, QMC [6]. In our previous investigation [5], we have applied this model in the Hartree approximation to derive the equation of state. From this we have deduced the density evolution of the quark condensate and of the QCD susceptibilities. This procedure insures the compatibility with the saturation properties of nuclear matter. Our conclusions were that the deviations of the condensate evolution with density from the linear behavior are mild and that there exists a convergence effect between the scalar and pseudoscalar susceptibilities, a conclusion already reached in Refs. [7,8].

The pion which does not contribute at the mean field level was ignored in this work. However the exchange of the scalar $s$ field between nucleons is not the only source of attraction. Beside the exchange of a scalar meson, the Van Der Waals type of forces, i.e., two-pion exchange with one or two intermediate Delta excitations, also contribute to the middle range attraction of the $N N$ potential and to the nuclear binding. Iterated pion exchange is also a source of attraction. It is even envisaged in a chiral theory that it could describe the bulk of the nuclear binding [9]. It is therefore natural to extend our previous descriptions restricted to the Hartree scheme so as to incorporate pion loops, which is the aim of the present article. When pion exchange forces are discussed one has also to introduce the other components of the spinisospin force, namely the short-range contribution embodied in the Landau Migdal parameters. We will also introduce rho exchange, which acts in the spin transverse channel. We will show that the role of both the short-range terms and the rho in the correlation energy is crucial. For what concerns the values of the Landau-Migdal parameters, years of experimental and theoretical investigations have sharpened our knowledge of these quantities. We will exploit here the most recent results, for which a survey is given in a recent article by Ichimura et al. [10]. We feel timely to revisit certain problems of nuclear physics and combine in these studies the experience accumulated in the pionic type of physics including chiral aspects and some results of QCD to have simultaneously a description of the nuclear binding and the evolution of chiral symmetry restoration, i.e., of the quark scalar density, and of the QCD susceptibilities.

In order to achieve this we will incorporate in a consistent way, on top of this mean field, the pion loop contribution to the energy, namely the pion Fock term and the pionic correlation energy, taking into account short-range correlation effects, within a RPA ring approximation [11]. In this way, by taking the successive derivatives of the grand potential with respect to the pion mass, we will obtain the full contribution to the evolution of the chiral condensate and the scalar susceptibility. In a recent work [12] we obtained the pionic piece of the scalar susceptibility by taking the derivative with respect to the quark mass (in practice to the pion mass) of the pion scalar density $\left\langle\Phi^{2}\right\rangle$, which is a component of the 
condensate of the medium. We showed that the medium effects affecting the scalar susceptibility are precisely the same that the ones governing the reshaping of the strength in the low invariant mass region in two-pion production experiments [13-15]. One purpose of the present paper is to provide a unified description of two quantities, i.e., the effect of the nuclear scalar field and of the pion loops in a way compatible with saturation properties of nuclear matter. The pionic contributions depend on the pionic polarization propagator i.e., the $p h$ (particle-hole) and $\Delta h$ (Delta-hole) Lindhardt functions. Although we start from a relativistic mean field theory we will evaluate these Lindhardt functions in a nonrelativistic framework for convenience since it is based on a well established phenomenology. It is interesting to point out that this approach where pion loops are incorporated on top of background scalar and vector mean-fields has some similarities with the approach of Ref. [16] based on a relativistic density functional. There are however important differences that will be discussed in Sec. IV.

Our article is organized as follows. Section II is devoted to the loop energy. After a brief summary of the results obtained in the Hartree scheme pion loops are introduced together with the other short-range components of the spin-isospin interaction. Section III discusses the QCD quantities, the quark condensate and the scalar susceptibility. Finally in Sec. IV numerical results are given and the results discussed.

\section{PION LOOP ENERGY}

\section{A. Summary of the mean field results without pion loop}

We start with the mean field Hamiltonian used in Ref. [5] supplemented by the free pion Hamiltonian:

$$
\begin{aligned}
H_{0}= & \int d \mathbf{r} \bar{N}\left(-i \vec{\gamma} \cdot \vec{\nabla}+M_{N}^{*}(s)+g_{\omega} \gamma^{0} \omega_{0}\right) N+V(s) \\
& -\frac{1}{2} m_{\omega}^{2} \omega_{0}^{2}+\sum_{\mathbf{q}, j} \omega_{q} b_{\mathbf{q} j}^{\dagger} b_{\mathbf{q} j},
\end{aligned}
$$

where $s$ is the chiral invariant field associated with the radius $S=f_{\pi}+s$ of the chiral circle. $V(s)=V_{0}(s)-c S$ is the vacuum potential which can be split into $V_{0}(s)=$ $(\lambda / 4)\left(\left(f_{\pi}+s\right)^{2}-v^{2}\right)^{2}$, responsible for spontaneous chiral symmetry breaking, and the explicit symmetry breaking piece, $-c S$, where $c=f_{\pi} m_{\pi}^{2}$. As usual in relativistic mean field theories we have added a coupling to an omega field $\omega_{0}$.

At the mean field level the energy density for symmetric nuclear matter is given by

$$
\frac{E_{0}}{V}=\varepsilon_{0}=\int \frac{4 d^{3} p}{(2 \pi)^{3}} \Theta\left(p_{F}-p\right) E_{p}^{*}(\bar{s})+V(\bar{s})+\frac{g_{\omega}^{2}}{2 m_{\omega}^{2}} \rho^{2}
$$

and $E_{p}^{*}(\bar{s})=\sqrt{p^{2}+M_{N}^{* 2}(\bar{s})}$ is the energy of an effective nucleon with the effective mass $M_{N}^{*}(\bar{s})$. The effective mass is sensitive to the effect of the nucleon susceptibility, $\kappa_{N S}$, which embeds the influence of the internal nucleon structure

$$
M_{N}^{*}(\bar{s})=M_{N}\left(1+\frac{\bar{s}}{f_{\pi}}\right)+\frac{1}{2} \kappa_{N S} \bar{s}^{2} .
$$

The expectation value, $\bar{S}=f_{\pi}+\bar{s}$, of the $S$ field plays the role of a chiral order parameter. It is obtained by minimizing the energy density: $\partial \varepsilon / \partial \bar{s}=g_{S}^{*} \rho_{S}+V^{\prime}(\bar{s})=0$ with the following expressions for the scalar density, $\rho_{S}$, and the scalar coupling constant $g_{S}^{*}$ :

$$
\rho_{S}=\int \frac{4 d^{3} p}{(2 \pi)^{3}} \Theta\left(p_{F}-p\right) \frac{M_{N}^{*}}{E_{p}^{*}}
$$

and

$$
g_{S}^{*}(\bar{s})=\frac{\partial M_{N}^{*}}{\partial \bar{s}}=\frac{M_{N}}{f_{\pi}}+\kappa_{N S} \bar{s} .
$$

The quantity $g_{S}=M_{N} / f_{\pi}$ is the scalar coupling constant of the model in the vacuum. Notice that the density dependence of $g_{S}^{*}$ arises entirely from the susceptibility term. Since the mean scalar field is negative and the sign of $\kappa_{N S}$ is positive, $g_{S}^{*}$ is a decreasing function of the density. The in-medium sigma mass is obtained as the second derivative of the energy density with respect to the order parameter: $m_{\sigma}^{* 2}=\partial^{2} \varepsilon / \partial \bar{s}^{2}=$ $V^{\prime \prime}(\bar{s})+\partial\left(g_{S}^{*} \rho_{S}\right) / \partial \bar{s}$.

In the free pion part of the Hamiltonian of Eq. (1), the operator $b_{\mathbf{q} j}^{\dagger}$ creates a free pion state with momentum $\mathbf{q}$ and isospin index $j$ and we we have $\omega_{q}=\sqrt{q^{2}+m_{\pi}^{2}}$ where $m_{\pi}$ is the pion mass. In fact in a more complete treatment including s-wave pion-nucleon coupling, according to Ref. [2], the pion mass should be replaced by an effective mass $m_{\pi}^{* 2}=m_{\pi}^{2}(1+$ $\bar{s} / f \pi)$. As in Ref. [17] we do not consider this effect since it is almost completely compensated by other contributions [18] and the pion mass remains stable in the nuclear medium [19].

\section{B. Inclusion of the pion-nucleon coupling}

We now include the usual derivative pion-nucleon interaction Hamiltonian:

$$
\begin{aligned}
H_{\pi N N} & =-\int d \mathbf{r} \frac{g_{A}}{f_{\pi}} \bar{N} \gamma^{\mu} \gamma_{5} \frac{\vec{\tau}}{2} \cdot \partial_{\mu} \vec{\Phi} N \\
& \simeq-\int d \mathbf{r} \frac{g_{A}}{2 f_{\pi}} N^{\dagger} \sigma \cdot \nabla \vec{\Phi} \cdot \vec{\tau} N,
\end{aligned}
$$

where the second form corresponds to the nonrelativistic limit of pure p-wave nature. It can be rewritten in a standard secondquantized form as

$$
\begin{aligned}
H_{\pi N N} & =\sum_{\mathbf{q}, j}\left(\frac{1}{2 \omega_{q} V}\right)^{1 / 2}\left(b_{\mathbf{q}, j}+b_{-\mathbf{q}, j}^{\dagger}\right) L_{j}(\mathbf{q}), \\
L_{j}(\mathbf{q}) & =\sum_{\alpha, \beta} c_{\beta}^{\dagger} c_{\alpha} V_{j}^{\beta \alpha}(\mathbf{q}), \\
V_{j}^{\beta \alpha}(\mathbf{q}, \omega) & =-i \frac{g_{A}}{2 f_{\pi}} v(\mathbf{q})\left\langle N: \beta\left|\boldsymbol{\sigma} \cdot \mathbf{q} \tau_{j} e^{i \mathbf{q} \cdot \mathbf{R}}\right| N: \alpha\right\rangle,
\end{aligned}
$$

where we have included phenomenologically a dipole $\pi N N$ form factor $v(\mathbf{q})$ and $V$ is the volume of the normalization box. Using Green's function techniques, the expectation value on the nuclear ground state $|0\rangle$ of this interacting Hamiltonian can be obtained according to [11]

$$
\left\langle H_{\pi N N}\right\rangle=3 V \int_{-\infty}^{+\infty} \frac{i d \omega}{(2 \pi)} \int \frac{d \mathbf{q}}{(2 \pi)^{3}} \frac{1}{\omega^{2}-\omega_{q}^{2}} \Pi_{L}(\omega, \mathbf{q}) .
$$


Here $\Pi_{L}(\omega, \mathbf{q})$ is the full spin-isospin polarization propagator defined by

$$
\begin{aligned}
\Pi_{L}(\omega, \mathbf{q})= & \int_{-\infty}^{+\infty} d\left(t-t^{\prime}\right) e^{i \omega\left(t-t^{\prime}\right)} \frac{(-i)}{V}\langle 0| T \\
& \times\left(L_{j}^{\dagger}(\mathbf{q}, t), L_{j}\left(\mathbf{q}, t^{\prime}\right)\right)|0\rangle \\
= & \frac{1}{V} \sum_{n} \frac{\left|\left\langle n\left|L_{j}(\mathbf{q})\right| 0\right\rangle\right|^{2}}{\omega-E_{n}+i \eta}-\frac{\left|\left\langle n\left|L_{j}^{\dagger}(\mathbf{q})\right| 0\right\rangle\right|^{2}}{\omega+E_{n}-i \eta} .
\end{aligned}
$$

We also include the Delta-hole states. In this case the operator $\boldsymbol{\sigma} . \mathbf{q} \tau_{j}$ is replaced by $\left(g_{\pi N \Delta} / g_{\pi N N}\right) \mathbf{S} \cdot \mathbf{q} T_{j}$ where the $S_{j}\left(T_{j}\right)$ are the standard spin (isospin) transition operators between spin (isospin) $1 / 2$ and $3 / 2$ states. For the ratio of coupling constants, we take the quark model value: $R_{N \Delta}=$ $\left(g_{\pi N \Delta} / g_{\pi N N}\right)=\sqrt{72 / 25}$.

\section{Inclusion of short-range correlation and rho meson exchange}

The expression (6) written above shows that the interaction energy of the pion cloud with matter is obtained by integrating the product of the full longitudinal spin-isospin polarization propagator (i.e., the nuclear response to a pion-like excitation) with the pion exchange potential. However, due to the correlated nature of the medium, the contact piece of the pion exchange potential should be treated with caution. In the part which concerns medium effects, this piece should be removed. This is achieved as usual by introducing in the spin-isospin channel a short-range two-body potential governed by Landau-Migdal $g^{\prime}$ parameters. The $N h-N h$ piece of the corresponding Hamiltonian writes

$$
\begin{aligned}
H_{\mathrm{sr}}= & \frac{1}{2} \sum_{\alpha \beta \gamma \delta} c_{\alpha}^{\dagger} c_{\beta}^{\dagger} c_{\delta} c_{\gamma}\left\langle\alpha \beta\left|V_{\mathrm{sr}}(1,2)\right| \gamma \delta\right\rangle \\
V_{\mathrm{sr}}(1,2)= & \int \frac{d \mathbf{q}}{(2 \pi)^{3}}\left(\frac{g_{A}}{2 f_{\pi}}\right)^{2} v^{2}(\mathbf{q}) g_{N N}^{\prime} \boldsymbol{\sigma}(1) \\
& \cdot \boldsymbol{\sigma}(2) \vec{\tau}(1) \cdot \vec{\tau}(2) e^{-i \mathbf{q} \cdot\left(\mathbf{R}_{1}-\mathbf{R}_{2}\right)}
\end{aligned}
$$

It is straightforward to extend this expression in order to include the $N h-\Delta h$ and the $\Delta h-\Delta h$ pieces. For this we replace $g_{N N}^{\prime}$ by $R_{N \Delta} g_{N \Delta}^{\prime}$ and $R_{N \Delta}^{2} g_{\Delta \Delta}^{\prime}$ where $R_{N \Delta}$ defined previously is the coupling constant ratio. In the numerical calculation we will use the following set of values suggested in Ref. [10], which takes into account the most recent data analysis: $g_{N N}^{\prime}=0.7, g_{N \Delta}^{\prime}=0.3, g_{\Delta \Delta}^{\prime}=0.5$, with clear deviations from universality as advocated in Ref. [10]. The spin-spin operator can be decomposed into a longitudinal piece and a transverse one. For this reason we also introduce the full transverse spin-isospin polarization propagator:

$$
\begin{aligned}
\Pi_{T}(\omega, \mathbf{q})= & \frac{1}{2} \int_{-\infty}^{+\infty} d\left(t-t^{\prime}\right) e^{i \omega\left(t-t^{\prime}\right)} \\
& \times \frac{(-i)}{V}\left\langle 0\left|T\left(\mathbf{T}_{j}^{\dagger}(\mathbf{q}, t), \mathbf{T}_{j}\left(\mathbf{q}, t^{\prime}\right)\right)\right| 0\right\rangle \\
= & \frac{1}{2 V} \sum_{n} \frac{\left|\left\langle n\left|\mathbf{T}_{j}(\mathbf{q})\right| 0\right\rangle\right|^{2}}{\omega-E_{n}+i \eta}-\frac{\left|\left\langle n\left|\mathbf{T}_{j}^{\dagger}(\mathbf{q})\right| 0\right\rangle\right|^{2}}{\omega+E_{n}-i \eta},
\end{aligned}
$$

where $\mathbf{T}_{j}$ is obtained from $L_{j}$ by replacing $\boldsymbol{\sigma} \cdot \mathbf{q}$ by $\boldsymbol{\sigma} \times \mathbf{q}$. The expectation value of the short-range potential is

$$
\begin{aligned}
\left\langle H_{\mathrm{sr}}\right\rangle= & \frac{3}{2} V \int_{-\infty}^{+\infty} \frac{i d \omega}{(2 \pi)} \int \frac{d \mathbf{q}}{(2 \pi)^{3}} g^{\prime}\left[\left(\Pi_{L}(\omega, \mathbf{q})\right.\right. \\
& \left.\left.+2 \Pi_{T}(\omega, \mathbf{q})\right)\right]-\frac{1}{2} \sum_{\alpha h}\left\langle\alpha h\left|V_{\mathrm{sr}}(1,2)\right| h \alpha\right\rangle,
\end{aligned}
$$

where the second term is introduced in order to remove the contribution for free nucleons. In the following, when this second term term is not explicitly written, this substraction is done implicitly. The first term $g^{\prime}\left(\Pi_{L}(\omega, \mathbf{q})+2 \Pi_{T}(\omega, \mathbf{q})\right)$ is written in a schematic form. The precise form involving the three Landau-Migdal parameters, $g_{N N}^{\prime}, g_{N \Delta}^{\prime}, g_{\Delta \Delta}^{\prime}$, will be given below in Sec. II D.

Finally, so as to obtain a more realistic description of the transverse spin-isospin channel we also introduce, beside the short-range piece, the $\rho$ meson through a $\rho N N$ Hamiltonian. In the nonrelativistic limit it has a structure similar to the $\pi N N$ one, once the $\sigma \cdot \mathbf{q}$ coupling is replaced by a $\sigma \times \mathbf{q}$ coupling. Its expectation value is

$$
\left\langle H_{\rho N N}\right\rangle=3 V \int_{-\infty}^{+\infty} \frac{i d \omega}{(2 \pi)} \int \frac{d \mathbf{q}}{(2 \pi)^{3}} \frac{2 C_{\rho}}{\omega^{2}-\Omega_{q}^{2}} \Pi_{T}(\omega, \mathbf{q})
$$

with $\Omega_{q}=\sqrt{q^{2}+m_{\rho}^{2}}$. We take $C_{\rho}=2$ which corresponds to the strong rho coupling [20].

\section{Calculation of the pion loop energy}

In order to calculate the pion loop energy we use the well-known charging formula method. For this purpose we introduce an auxiliary Hamiltonian which depends on one strength parameter $\lambda$ :

$$
H(\lambda)=H_{0}+\lambda\left(H_{\pi N N}+H_{\rho N N}\right)+\lambda^{2} H_{\mathrm{sr}} .
$$

$H(\lambda)$ coincides with the original Hamiltonian for $\lambda=1$. The charging formula allows the evaluation of the modification of the ground state energy with respect to the ground state energy $E_{0}$ of the $H_{0}$ Hamiltonian:

$$
\begin{aligned}
E_{\text {loop }}= & E-E_{0} \equiv V \varepsilon_{\text {loop }} \\
= & \frac{3}{2} V \int_{-\infty}^{+\infty} \frac{i d \omega}{(2 \pi)} \int \frac{d \mathbf{q}}{(2 \pi)^{3}} \int_{0}^{1} \frac{d \lambda}{\lambda} \\
& \times\left(\left[V_{L}(\omega, \mathbf{q}) \Pi_{L}(\omega, \mathbf{q} ; \lambda)\right]\right. \\
& \left.+2\left[V_{T}(\omega, \mathbf{q}) \Pi_{T}(\omega, \mathbf{q} ; \lambda)\right]\right) .
\end{aligned}
$$

The dependence on $\lambda$ of the full polarization propagators can be obtained by systematically multiplying the coupling $g_{A} / f_{\pi}$ by $\lambda$. For instance there appears a net $\lambda^{2}$ factor in front of each first order Lindhardt function appearing in the RPA expression of the full polarization propagators. The quantities $V_{L, T}(\omega, \mathbf{q})$ are the full energy dependent residual interactions in the longitudinal and transverse spin-isospin channels. In the 
$N h-N h$ sector, they write

$$
\begin{aligned}
& V_{L N N}(\omega, \mathbf{q})=g_{N N}^{\prime}+\frac{q^{2}}{\omega^{2}-\omega_{q}^{2}}, \\
& V_{T N N}(\omega, \mathbf{q})=g_{N N}^{\prime}+C_{\rho} \frac{q^{2}}{\omega^{2}-\Omega_{q}^{2}}
\end{aligned}
$$

with identical expressions in the $N h-\Delta h$ and the $\Delta h-\Delta h$ channels once the relevant $g^{\prime}$ parameters have been incorporated. Again the expression (13) written above is schematic and the full formula calculated in the RPA ring approximation is given just below in Eqs. (16) and (17). The $\lambda$ integration can be done analytically to obtain the loop energy density $\varepsilon_{\text {loop }}$ :

$$
\varepsilon_{\text {loop }}=\varepsilon_{L}+\varepsilon_{T}
$$

with

$$
\begin{aligned}
\varepsilon_{L}= & \frac{3}{2} \int \frac{i d \omega d \mathbf{q}}{(2 \pi)^{4}}\left[-\ln \left(1-V_{L N N} \Pi_{N}^{0}-V_{L N \Delta} \Pi_{\Delta}^{0}\right.\right. \\
& \left.\left.-\left(V_{L N \Delta}^{2}-V_{L N N} V_{L \Delta \Delta}\right) \Pi_{N}^{0} \Pi_{\Delta}^{0}\right)\right], \\
\varepsilon_{T}= & 3 \int \frac{i d \omega d \mathbf{q}}{(2 \pi)^{4}}\left[-\ln \left(1-V_{T N N} \Pi_{N}^{0}-V_{T N \Delta} \Pi_{\Delta}^{0}\right.\right. \\
& \left.\left.-\left(V_{T N \Delta}^{2}-V_{T N N} V_{T \Delta \Delta}\right) \Pi_{N}^{0} \Pi_{\Delta}^{0}\right)\right] .
\end{aligned}
$$

$\Pi_{N}^{0}$ is the $N h$ Lindhardt function which is calculated nonrelativistically. With the single particle energy, $\varepsilon_{p}=p^{2} / 2 M^{*}$ where $M^{*}$ is the $s$ dependent nucleon effective mass calculated at the mean-field level, $\Pi_{N}^{0}$ has the familiar form

$$
\begin{aligned}
\Pi_{N}^{0}(\omega, \mathbf{q})= & 4\left(\frac{g_{A}}{2 f_{\pi}}\right)^{2} v^{2}(\mathbf{q}) \int \frac{d \mathbf{p}}{(2 \pi)^{3}} \\
& \times\left(\frac{\Theta\left(p_{F}-p\right) \Theta\left(|\mathbf{p}+\mathbf{q}|-p_{F}\right)}{\omega-\epsilon_{N \mathbf{p q}}+i \eta}\right. \\
& \left.-\frac{\Theta\left(p_{F}-p\right) \Theta\left(|\mathbf{p}+\mathbf{q}|-p_{F}\right)}{\omega+\epsilon_{N \mathbf{p q}}-i \eta}\right) .
\end{aligned}
$$

$\epsilon_{N \mathbf{p q}}=\epsilon_{\mathbf{p}+\mathbf{q}}-\epsilon_{\mathbf{p}}$ is the energy of the $p h$ excitation. The corresponding $\Delta h$ Lindhardt function, $\Pi_{\Delta}^{0}$ is also calculated nonrelativistically. Introducing also an effective $\Delta$ mass, we make the natural assumption that the in-medium shift of the nucleon and delta masses in presence of the nuclear scalar field are identical. The $\Delta h$ energy is thus $\epsilon_{\Delta \mathbf{p q}}=\epsilon_{\Delta, \mathbf{p}+\mathbf{q}}-\epsilon_{\mathbf{p}}$ with $\epsilon_{\Delta, \mathbf{p}}=M_{\Delta}-M_{N}+p^{2} / 2 M_{\Delta}^{*}$ and $\Pi_{\Delta}^{0}$ writes

$$
\begin{aligned}
\Pi_{\Delta}^{0}(\omega, \mathbf{q})= & \frac{16}{9}\left(\frac{g_{A}}{2 f_{\pi}} R_{N \Delta}\right)^{2} v^{2}(\mathbf{k}) \int \frac{d \mathbf{p}}{(2 \pi)^{3}} \Theta\left(p_{F}-p\right) \\
& \times\left(\frac{1}{\omega-\epsilon_{\Delta \mathbf{p q}}+i \eta}-\frac{1}{\omega+\epsilon_{\Delta \mathbf{p q}}}\right)
\end{aligned}
$$

This loop energy can be split in a piece of mean-field nature (containing the Fock term) and a genuine correlation energy.
For instance we have for the longitudinal piece

$$
\begin{aligned}
\varepsilon_{L}= & \varepsilon_{L}^{M F}+\varepsilon_{L}^{\text {Corr }} \\
\varepsilon_{L}^{M F}= & \frac{3}{2} \int \frac{i d \omega d \mathbf{q}}{(2 \pi)^{4}}\left[V_{L N N} \Pi_{N}^{0}+V_{L N \Delta} \Pi_{\Delta}^{0}\right] \\
\varepsilon_{L}^{\text {Corr }}= & \frac{3}{2} \int \frac{i d \omega d \mathbf{q}}{(2 \pi)^{4}}\left[-\ln \left(1-V_{L N N} \Pi_{N}^{0}-V_{L N \Delta} \Pi_{\Delta}^{0}\right.\right. \\
& \left.-\left(V_{L N \Delta}^{2}-V_{L N N} V_{L \Delta \Delta}\right) \Pi_{N}^{0} \Pi_{\Delta}^{0}\right) \\
& \left.-V_{L N N} \Pi_{N}^{0}-V_{L N \Delta} \Pi_{\Delta}^{0}\right] .
\end{aligned}
$$

By performing the energy integration with a Wick rotation, $\varepsilon_{L}^{M F}$ can be expressed as follows:

$$
\varepsilon_{L}^{M F}=\int \frac{4 d \mathbf{p}}{(2 \pi)^{3}} \Theta\left(p_{F}-p\right) \Sigma_{\pi}(\mathbf{p})+\varepsilon_{L}^{\text {Fock }} .
$$

$\Sigma_{\pi}(\mathbf{p})$ is the pionic contribution to the nucleon self-energy:

$$
\begin{aligned}
\Sigma_{\pi}(\mathbf{p})= & -\frac{3}{2}\left(\frac{g_{A}}{2 f_{\pi}}\right)^{2} \int \frac{d \mathbf{q}}{(2 \pi)^{3}} \mathbf{q}^{2} v^{2}(\mathbf{q}) \\
& \times\left(\frac{1}{\omega_{q}} \frac{1}{\omega_{q}+\epsilon_{N \mathbf{p q}}}+\frac{4 R_{N \Delta}^{2}}{9} \frac{1}{\omega_{q}} \frac{1}{\omega_{q}+\epsilon_{\Delta \mathbf{p q}}}\right) .
\end{aligned}
$$

In principle medium effects are present in this quantity since the $N h$ and $\Delta h$ energies depend on the effective nucleon and delta masses but in practice we ignore these effects. The momentum dependence of the nucleon self-energy is linked to the treatment of the vacuum nucleon which is not the purpose of this paper. Hence we ignore it. At zero momentum the self-energy $\Sigma_{\pi} \equiv \Sigma_{\pi}(\mathbf{p}=0)$ is similar to the model calculation one used to fit lattice data by Thomas et al. [21], although we do not make a static approximation. In our calculation we will choose a dipole form factor, with a cutoff $\Lambda=0.98 \mathrm{GeV}$, such that the resulting contribution to the free nucleon sigma term, $\sigma_{N}^{(\pi)}$, the expression of which being given in Sec. III A, is $\sigma_{N}^{(\pi)}=21.5 \mathrm{MeV}$, in agreement with previous determinations [21-23]. The corresponding pion cloud self-energy is $\Sigma_{\pi}=-420 \mathrm{MeV}$.

The Fock term is

$$
\begin{aligned}
\varepsilon_{L}^{\text {Fock }}= & -\frac{3}{2}\left(\frac{g_{A}}{2 f_{\pi}}\right)^{2} \int \frac{d \mathbf{q}}{(2 \pi)^{3}} v^{2}(\mathbf{q}) \\
& \times \int \frac{4 d \mathbf{p}}{(2 \pi)^{3}} \Theta\left(p_{F}-p\right) \Theta\left(p_{F}-|\mathbf{p}+\mathbf{q}|\right) \\
& \times\left(g_{N N}^{\prime}-\frac{\mathbf{q}^{2}}{\omega_{q}\left(\omega_{q}+\epsilon_{N \mathbf{p q}}\right)}\right)
\end{aligned}
$$

Notice that this Fock term includes a retardation effect through the presence of the particle-hole energy $\epsilon_{N \mathbf{p q}}$. In practice the effect is numerically small and we take it as its average Fermi sea value: $\epsilon_{N \mathbf{p q}}=\epsilon_{N q}=q^{2} / 2 M_{N}$, ignoring the in-medium modification of the nucleon mass. In that way the Fock energy does not depend on $\bar{s}$, a feature which simplifies the calculation of the scalar susceptibility. Also notice that, at variance with other works, see, e.g., Ref. [24], we keep the form factor in the Fock term. The Fock term relative to the transverse channel is 
obtained in a similar way:

$$
\begin{aligned}
\varepsilon_{T}^{\mathrm{Fock}}= & -3\left(\frac{g_{A}}{2 f_{\pi}}\right)^{2} \int \frac{d \mathbf{q}}{(2 \pi)^{3}} v^{2}(\mathbf{q}) \\
& \times \int \frac{4 d \mathbf{p}}{(2 \pi)^{3}} \Theta\left(p_{F}-p\right) \Theta\left(p_{F}-|\mathbf{p}+\mathbf{q}|\right) \\
& \times\left(g_{N N}^{\prime}-\frac{C_{\rho} \mathbf{q}^{2}}{\Omega_{q}\left(\Omega_{q}+\epsilon_{N \mathbf{p q}}\right)}\right) .
\end{aligned}
$$

Finally the correlation energy is also calculated using a Wick rotation for the $\omega$ energy integration. The numerical results will be given in Sec. IV.

\section{THE CHIRAL CONDENSATE AND THE SCALAR SUSCEPTIBILITY}

\section{A. Direct calculation}

In this first approach the in-medium quark condensate is evaluated directly from the expectation value of chiral symmetry breaking piece of the Hamiltonian:

$$
\begin{aligned}
\left\langle H_{\chi S B}\right\rangle & \equiv V 2 m\langle\bar{q} q\rangle \simeq V\left\langle\frac{1}{2} m_{\pi}^{2} \Phi^{2}-c S\right\rangle \\
& \rightarrow\langle\bar{q} q\rangle \simeq\langle\bar{q} q\rangle_{\mathrm{vac}}\left(1-\frac{\left\langle\Phi^{2}\right\rangle}{2 f_{\pi}^{2}}+\frac{\bar{s}}{f_{\pi}}\right),
\end{aligned}
$$

where the GOR (Gell-Mann-Oakes-Renner) relation has been used to obtain the second equation. The pion scalar density can be directly calculated from the in-medium pion propagator $[12,25]$ :

$$
\begin{aligned}
\left\langle\Phi^{2}\right\rangle & =3 \int \frac{i d \omega d \mathbf{q}}{(2 \pi)^{4}}\left[D_{\pi}-D_{0 \pi}\right](\omega, \mathbf{q}) \\
& =3 \int \frac{i d \omega d \mathbf{q}}{(2 \pi)^{4}}\left[D_{\pi} D_{0 \pi}(\omega, \mathbf{q}) \mathbf{q}^{2} \tilde{\Pi}^{0}(\omega, \mathbf{q})\right] .
\end{aligned}
$$

$D_{\pi}=\left(\omega^{2}-\omega_{q}^{2}-\mathbf{q}^{2} \tilde{\Pi}^{0}\right)^{-1}$ is the full pion propagator, $\mathbf{q}^{2} \tilde{\Pi}^{0}$ is the irreducible pion self-energy with

$$
\tilde{\Pi}^{0}=\frac{\Pi_{N}^{0}+\Pi_{\Delta}^{0}+\left(2 g_{N \Delta}^{\prime}-g_{N N}^{\prime}-g_{\Delta \Delta}^{\prime}\right) \Pi_{N}^{0} \Pi_{\Delta}^{0}}{1-\left(g_{N N}^{\prime} \Pi_{N}^{0}+g_{\Delta \Delta}^{\prime} \Pi_{\Delta}^{0}+\left(g_{N \Delta}^{\prime 2}-g_{N N}^{\prime} g_{\Delta \Delta}^{\prime}\right) \Pi_{N}^{0} \Pi_{\Delta}^{0}\right)} .
$$

It is possible to show that the formal expression of the pion scalar density can be obtained as the derivative with respect to $m_{\pi}^{2}$ of the pionic energy density $\varepsilon_{L}$ given by Eq. (16):

$$
\frac{\left\langle\Phi^{2}\right\rangle}{2}=\frac{\partial \varepsilon_{L}}{\partial m_{\pi}^{2}} \quad \text { or equivalently } \quad-\langle\bar{q} q\rangle_{\mathrm{vac}} \frac{\left\langle\Phi^{2}\right\rangle}{2 f_{\pi}^{2}}=\frac{1}{2} \frac{\partial \varepsilon_{L}}{\partial m}
$$

which is nothing but the Feynman-Hellman theorem applied to the pion loop contribution. As in the previous section we decompose $\varepsilon_{L}$ into a free nucleon contribution, a Fock contribution and a correlation piece, with a similar decomposition for $\left\langle\Phi^{2}\right\rangle$ :

$$
\begin{aligned}
\frac{\left\langle\Phi^{2}\right\rangle}{2} & =\rho \frac{\partial \Sigma_{\pi}}{\partial m_{\pi}^{2}}+\frac{\partial \varepsilon_{L}^{\text {Fock }}}{\partial m_{\pi}^{2}}+\frac{\partial \varepsilon_{L}^{\text {Corr }}}{\partial m_{\pi}^{2}} \\
& \equiv \rho \frac{\sigma_{N}^{(\pi)}}{m_{\pi}^{2}}+\frac{\left\langle\Phi^{2}\right\rangle^{\text {Fock }}}{2}+\frac{\left\langle\Phi^{2}\right\rangle^{\text {Corr }}}{2}
\end{aligned}
$$

The pionic contribution to the pion-nucleon sigma term is

$$
\begin{aligned}
\sigma_{N}^{(\pi)}= & \frac{3}{2}\left(\frac{g_{A}}{2 f_{\pi}}\right)^{2} m_{\pi}^{2} \int \frac{d \mathbf{q}}{(2 \pi)^{3}} \frac{\mathbf{q}^{2} v^{2}(\mathbf{q})}{2 \omega_{q}^{2}} \\
& \times\left[\frac{1}{\omega_{q}} \frac{1}{\omega_{q}+\epsilon_{q}}+\frac{1}{\left(\omega_{q}+\epsilon_{q}\right)^{2}}\right. \\
& \left.+\frac{4 R_{N \Delta}^{2}}{9}\left(\frac{1}{\omega_{q}} \frac{1}{\omega_{q}+\epsilon_{\Delta q}}+\frac{1}{\left(\omega_{q}+\epsilon_{\Delta q}\right)^{2}}\right)\right] .
\end{aligned}
$$

The Fock term contribution has the following explicit form:

$$
\begin{aligned}
\frac{\left\langle\Phi^{2}\right\rangle^{\text {Fock }}}{2}= & -\frac{3}{2}\left(\frac{g_{A}}{2 f_{\pi}}\right)^{2} \rho \int_{0}^{2 p_{F}} \frac{d \mathbf{q}}{(2 \pi)^{3}} \frac{\mathbf{q}^{2} v^{2}(\mathbf{q})}{2 \omega_{q}^{2}\left(\omega_{q}+\epsilon_{q}\right)} \\
& \times\left(1-\frac{3 q}{4 p_{F}}+\frac{q^{3}}{16 p_{F}^{3}}\right)\left(\frac{1}{\omega_{q}}+\frac{1}{\left(\omega_{q}+\epsilon_{q}\right)}\right) .
\end{aligned}
$$

Finally the correlation piece is obtained by removing the leading order piece in Eq. (26):

$$
\frac{\left\langle\Phi^{2}\right\rangle^{\text {Corr }}}{2}=\frac{3}{2} \int \frac{i d \omega d \mathbf{q}}{(2 \pi)^{4}} \mathbf{q}^{2}\left[D_{\pi} D_{0 \pi} \tilde{\Pi}^{0}-D_{0 \pi}^{2} \Pi^{0}\right](\omega, \mathbf{q}) .
$$

In the next subsection we will evaluate the condensate from the equation of state (grand potential) and the FeynmanHellman theorem. It leads essentially to the same result with a modification coming from the fact that we will have to face the problem of the Lorentz nature of the in-medium effects of the pion loop energy.

\section{B. The quark condensate from the equation of state}

The pion cloud contribution has been calculated within the standard non relativistic framework. One reason is the basis of the pion-nucleus phenomenology. However some care should be taken when this pion cloud is included on top of the relativistic mean field used to describe the coupling of the scalar field to nucleons. For free nucleons the pionic self-energy $\Sigma_{\pi}$ has to be of scalar nature and thus it adds to the bare nucleon mass: $M_{N}=M_{0}+\Sigma_{\pi}$. For what concerns the in-medium contribution to the energy we do not know if it is of scalar or vector nature or a mixture of both. Here we assume its vector nature and add it to the energy density. This assumption affects contributions arising from the pionic interaction between nucleons where it amounts to distinguishing between scalar and vector densities. In practice the effect of this difference is numerically quite small. For the free nucleon part instead this distinction has a non-negligible 
effect. The energy density thus writes

$$
\begin{aligned}
\frac{E}{V}= & \varepsilon=\int \frac{4 d^{3} p}{(2 \pi)^{3}} \Theta\left(p_{F}-p\right) E_{p}^{*}(\bar{s}) \\
& +V(\bar{s})+\frac{g_{\omega}^{2}}{2 m_{\omega}^{2}} \rho^{2}+\varepsilon^{F C},
\end{aligned}
$$

where $\varepsilon^{F C}=\varepsilon_{L}^{\text {Fock }}+\varepsilon_{T}^{\text {Fock }}+\varepsilon_{L}^{\text {Corr }}+\varepsilon_{T}^{\text {Corr }}$ summarizes the loop energy density from the Fock term and correlations (pion+rho+short-range) which can be decomposed into a longitudinal piece (pion+short-range) and a transverse piece (rho+short-range). The mean field $\bar{s}$ is obtained by minimizing the energy density before the incorporation of the loop energy or, said differently, ignoring the $\bar{s}$ dependance of $\varepsilon^{F C}$. This is actually true for the Fock term and this approximation has a small numerical incidence on the evolution of the condensate with inclusion of the correlation energy. It also follows from the independence on $\bar{s}$ of $\epsilon^{F C}$ that the in-medium sigma mass remains the same as in the mean-field case:

$$
m_{\sigma}^{* 2}=\frac{\partial^{2} \varepsilon}{\partial \bar{s}^{2}}=V^{\prime \prime}(\bar{s})+\kappa_{N S} \rho_{S}+g_{S}^{*} \frac{\partial \rho_{S}^{*}}{\partial \bar{s}} .
$$

As discussed in Ref. [5] the scalar susceptibility term (second term in $\kappa_{N S}$ on the r.h.s of Eq. (34)) counterbalances the effect of the first term, the in-medium chiral dropping of the sigma mass. The last term actually corresponds to the nuclear response associated with $N \bar{N}$ excitation. In practice it is small and it can be omitted [8].

We will derive the in-medium chiral condensate and the QCD scalar susceptibility from the equation of state since they are related to the first and second derivatives of the grand potential with respect to the quark mass $m$ at constant chemical potential $\mu$. The baryonic chemical potential is obtained as

$$
\mu=\frac{\partial \varepsilon}{\partial \rho}=E_{F}^{*}+\frac{g_{\omega}^{2}}{m_{\omega}^{2}} \rho+\frac{\partial \varepsilon^{F C}}{\partial \rho}
$$

with

$$
E_{F}^{*}=\sqrt{p_{F}^{2}+M_{N}^{* 2}(\bar{s})} .
$$

In order to obtain this result we have taken into account only the explicit density dependence on the density $\rho$, i.e., keeping $\bar{s}$ constant since $\partial \varepsilon / \partial \bar{s}=0$. One deduces that the baryonic density is controlled by the chemical potential according to

$$
\rho=\int \frac{4 d^{3} p}{(2 \pi)^{3}} \Theta\left(\mu-E_{p}^{*}-\frac{g_{\omega}^{2}}{m_{\omega}^{2}} \rho-\frac{\partial \varepsilon^{F C}}{\partial \rho}\right)
$$

while the scalar density writes

$$
\rho_{S}=\int \frac{4 d^{3} p}{(2 \pi)^{3}} \frac{M_{N}^{*}}{E_{p}^{*}} \Theta\left(\mu-E_{p}^{*}-\frac{g_{\omega}^{2}}{m_{\omega}^{2}} \rho-\frac{\partial \varepsilon^{F C}}{\partial \rho}\right) .
$$

The grand potential, which is obtained through a Legendre transform, can be written in the following form:

$$
\begin{aligned}
\omega(\mu) & =\varepsilon-\mu \rho \\
& =\int \frac{4 d^{3} p}{(2 \pi)^{3}}\left(E_{p}^{*}+\frac{g_{\omega}^{2}}{m_{\omega}^{2}} \rho+\frac{\partial \varepsilon^{F C}}{\partial \rho}-\mu\right)
\end{aligned}
$$

$$
\begin{aligned}
& \times \Theta\left(\mu-E_{p}^{*}-\frac{g_{\omega}^{2}}{m_{\omega}^{2}} \rho-\frac{\partial \varepsilon^{F C}}{\partial \rho}\right) \\
& +V(s)-\frac{g_{\omega}^{2}}{2 m_{\omega}^{2}} \rho^{2}+\varepsilon^{F C}-\rho \frac{\partial \varepsilon^{F C}}{\partial \rho} .
\end{aligned}
$$

For the derivation of the condensate and of the susceptibility we have to specify the symmetry breaking parameter, which in QCD is the quark mass. In the context of this model it is the quantity $c=f_{\pi} m_{\pi}^{2}$ which enters the symmetry breaking piece of the potential. In the application of the Feynman-Hellman theorem we use the explicit expression of $\partial c / \partial m$ given by the model to leading order in the quark mass $m$, i.e., $\partial c / \partial m=$ $-2\langle\bar{q} q\rangle_{\mathrm{vac}} / f_{\pi}$. As mentioned previously, in the calculation of the derivative we only keep the explicit dependence on $c$ :

$$
\begin{aligned}
\langle\bar{q} q\rangle & =\frac{1}{2}\left(\frac{\partial \omega}{\partial m}\right)_{\mu}=\frac{1}{2} \frac{\partial c}{\partial m}\left(\frac{\partial \omega}{\partial c}\right)_{\mu} \\
& \simeq-\frac{\langle\bar{q} q\rangle_{\mathrm{vac}}}{f_{\pi}}\left(-\bar{S}+\rho_{S}\left(\frac{\partial M_{N}}{\partial c}\right)_{\bar{S}}+\frac{\partial \varepsilon_{L}^{F C}}{\partial c}\right) \\
& \simeq\langle\bar{q} q\rangle_{\mathrm{vac}}\left(\frac{\bar{S}}{f_{\pi}}-\rho_{S} \frac{m_{\pi}^{2}}{c} \frac{\partial \Sigma_{\pi}}{\partial m_{\pi}^{2}}-\frac{m_{\pi}^{2}}{c} \frac{\partial \varepsilon_{L}^{F C}}{\partial m_{\pi}^{2}}\right) \\
& =\langle\bar{q} q\rangle_{\mathrm{vac}}\left(1+\frac{\bar{s}}{f_{\pi}}-\frac{\left\langle\Phi^{2}\right\rangle}{2 f_{\pi}^{2}}\right) .
\end{aligned}
$$

In the last line we have grouped the pionic contribution in a pion scalar density given by

$$
\frac{\left\langle\Phi^{2}\right\rangle}{2}=\rho_{S} \frac{\sigma_{N}^{(\pi)}}{m_{\pi}^{2}}+\frac{\left\langle\Phi^{2}\right\rangle^{\text {Fock }}}{2}+\frac{\left\langle\Phi^{2}\right\rangle^{\text {Corr }}}{2} .
$$

Notice that the pion scalar density deviates from the fully nonrelativistic one of Eq. (26) since the leading order term behaves like $\rho_{S}$ in place of $\rho$. To leading order in density we recover, as expected, the well-known result:

$$
\begin{aligned}
\langle\bar{q} q\rangle & =\langle\bar{q} q\rangle_{\mathrm{vac}}\left(1-\frac{\sigma_{N} \rho}{f_{\pi}^{2} m_{\pi}^{2}}\right), \\
\sigma_{N} & =\sigma_{N}^{(\pi)}+\sigma_{N}^{(\sigma)}, \\
\sigma_{N}^{(\sigma)} & =f_{\pi} g_{S} \frac{m_{\pi}^{2}}{m_{\sigma}^{2}} .
\end{aligned}
$$

\section{The scalar susceptibility}

The in-medium scalar susceptibility is obtained as a derivative at fixed $\mu$ of the chiral condensate given in Eq. (39). The dependence of the pion scalar density on the symmetry breaking parameter $\left(c\right.$ or $m_{\pi}$ ) receives two contributions: one comes from the explicit dependence on $c$ and the other from the implicit dependence since, at fixed $\mu$, the density $\rho$ depends on $c$ according to Eq. (36). Hence the scalar susceptibility takes the form

$$
\begin{aligned}
\chi_{S} & =\left(\frac{\partial\langle\bar{q} q\rangle}{\partial m}\right)_{\mu} \\
& =-\frac{1}{2}\left(\frac{\partial c}{\partial m}\right)^{2}\left[\left(\frac{\partial \bar{S}}{\partial c}\right)_{\mu}-\frac{1}{2 f_{\pi}}\left(\frac{\partial\left\langle\Phi^{2}\right\rangle}{\partial c}\right)_{\mu}\right]
\end{aligned}
$$




$$
\begin{aligned}
\simeq & -2 \frac{\langle\bar{q} q\rangle_{\mathrm{vac}}^{2}}{f_{\pi}^{2}}\left[\left(\frac{\partial \bar{S}}{\partial c}\right)_{\mu}-\frac{1}{2 f_{\pi}}\left(\frac{\partial\left\langle\Phi^{2}\right\rangle}{\partial \rho}\right)_{\mu}\left(\frac{\partial \rho}{\partial c}\right)_{\mu}\right. \\
& \left.-\frac{m_{\pi}^{2}}{2 f_{\pi} c}\left(\frac{\partial\left\langle\Phi^{2}\right\rangle}{\partial m_{\pi}^{2}}\right)\right] \\
\equiv & \chi_{S}^{\text {nuclear }}+\chi_{S}^{\text {pion loop }} .
\end{aligned}
$$

We have divided $\chi_{S}$ in two components. The first one which is the sum of the first two terms contains the coupling of the scalar quark density fluctuations to the nuclear $p h$ excitations. For this reason we denote it $\chi_{S}^{\text {nuclear: }}$

$$
\begin{aligned}
\chi_{S}^{\text {nuclear }}= & -2 \frac{\langle\bar{q} q\rangle_{\text {vac }}^{2}}{f_{\pi}^{2}}\left[\left(\frac{\partial \bar{S}}{\partial c}\right)_{\mu}\right. \\
& \left.-\frac{1}{2 f_{\pi}}\left(\frac{\partial\left\langle\Phi^{2}\right\rangle}{\partial \rho}\right)_{\mu}\left(\frac{\partial \rho}{\partial c}\right)_{\mu}\right] .
\end{aligned}
$$

The second one, $\chi_{S}^{\text {pion loop }}$, of purely pionic nature, comes from the explicit dependence on $m_{\pi}$ of the pion scalar density $\left\langle\Phi^{2}\right\rangle$. The latter can be written as

$$
\begin{aligned}
\chi_{S}^{\text {pion loop }} & =2 \frac{\langle\bar{q} q\rangle_{\text {vac }}^{2}}{f_{\pi}^{2}} \frac{1}{2 f_{\pi}^{2}} \frac{\partial\left\langle\Phi^{2}\right\rangle}{\partial m_{\pi}^{2}} \\
& =\rho_{S} \chi_{N S}^{(\pi)}+\chi_{S}^{\text {pion-Fock }}+\chi_{S}^{\text {pion-Corr }} .
\end{aligned}
$$

The pionic contribution to the nucleon scalar susceptibility $\chi_{N S}^{(\pi)}$ is obtained from the derivative of the pion cloud contribution to the sigma commutator [Eq. (30)]:

$$
\begin{aligned}
\chi_{N S}^{(\pi)}= & -\frac{\langle\bar{q} q\rangle_{\mathrm{vac}}^{2}}{f_{\pi}^{4}} \frac{3}{2}\left(\frac{g_{A}}{2 f_{\pi}}\right)^{2} \int \frac{d \mathbf{q}}{(2 \pi)^{3}} \frac{\mathbf{q}^{2} v^{2}(\mathbf{q})}{2 \omega_{q}^{3}}\left[\frac{3}{\omega_{q}^{2}\left(\omega_{q}+\epsilon_{q}\right)}\right. \\
& +\frac{3}{\omega_{q}\left(\omega_{q}+\epsilon_{q}\right)^{2}}+\frac{2}{\left(\omega_{q}+\epsilon_{q}\right)^{3}}+\frac{4 R_{N \Delta}^{2}}{9} \\
& \times\left(\frac{3}{\omega_{q}^{2}\left(\omega_{q}+\epsilon_{\Delta q}\right)}+\frac{3}{\omega_{q}\left(\omega_{q}+\epsilon_{\Delta q}\right)^{2}}\right. \\
& \left.\left.+\frac{2}{\left(\omega_{q}+\epsilon_{\Delta q}\right)^{3}}\right)\right] .
\end{aligned}
$$

The Fock term contribution is obtained from Eq. (31):

$$
\begin{aligned}
\chi_{S}^{\text {pion-Fock }}= & \frac{\langle\bar{q} q\rangle_{\mathrm{vac}}^{2}}{f_{\pi}^{4}} \frac{3}{2}\left(\frac{g_{A}}{2 f_{\pi}}\right)^{2} \rho \int_{0}^{2 p_{F}} \frac{d \mathbf{q}}{(2 \pi)^{3}} \\
& \times \frac{\mathbf{q}^{2} v^{2}(\mathbf{q})}{2 \omega_{q}^{3}\left(\omega_{q}+\epsilon_{q}\right)}\left(1-\frac{3 q}{4 p_{F}}+\frac{q^{3}}{16 p_{F}^{3}}\right) \\
& \times\left(\frac{3}{\omega_{q}^{2}\left(\omega_{q}+\epsilon_{q}\right)}+\frac{3}{\omega_{q}\left(\omega_{q}+\epsilon_{q}\right)^{2}}\right. \\
& \left.+\frac{2}{\left(\omega_{q}+\epsilon_{q}\right)^{3}}\right) .
\end{aligned}
$$

Finally the correlation piece is obtained from Eq. (32):

$$
\begin{aligned}
\chi_{S}^{\text {pion-Corr }}= & 2 \frac{\langle\bar{q} q\rangle_{\mathrm{vac}}^{2}}{f_{\pi}^{4}} \frac{3}{2} \int \frac{i d \omega d \mathbf{q}}{(2 \pi)^{4}} \mathbf{q}^{2} \\
& \times\left[\left(D_{\pi}^{2} D_{0 \pi}+D_{\pi} D_{0 \pi}^{2}\right) \tilde{\Pi}^{0}-2 D_{0 \pi}^{3} \Pi^{0}\right](\omega, \mathbf{q}) .
\end{aligned}
$$

In the evaluation of the nuclear contribution, $\chi_{S}^{\text {nuclear }}$, the derivative $(\partial \bar{S} / \partial c)_{\mu}$ is obtained by taking the derivative of the minimization equation with respect to the parameter $c$ :

$$
\begin{aligned}
m_{\sigma}^{* 2}\left(\frac{\partial \bar{S}}{\partial c}\right)_{\mu}= & 1-g_{S}^{*} \frac{M_{N}^{*}}{E_{F}^{*}} \Pi_{0}(0)\left[g_{S}^{*}\left(\frac{\partial \bar{S}}{\partial c}\right)_{\mu}\right. \\
& \left.+\frac{E_{F}^{*}}{M_{N}^{*}} X^{(\pi)}+V_{V}\left(\frac{\partial \rho}{\partial c}\right)_{\mu}\right]
\end{aligned}
$$

with the auxiliary quantities

$$
\begin{aligned}
X^{(\pi)} & =\frac{1}{2 f_{\pi}} \frac{\partial\left\langle\Phi^{2}\right\rangle^{F C}}{\partial \rho}+\frac{M_{N}^{*}}{E_{F}^{*}} \frac{\sigma_{N}^{(\pi)}}{f_{\pi} m_{\pi}^{2}}, \\
V_{V} & =\frac{E_{F}^{*}}{M_{N}^{*}}\left(\frac{g_{\omega}^{2}}{m_{\omega}^{2}}+\frac{\partial^{2} \varepsilon^{F C}}{\partial \rho^{2}}\right)
\end{aligned}
$$

and with $\Pi_{0}(0)=-2 M_{N}^{*} p_{F} / \pi^{2}$, which is the nonrelativistic free Fermi gas particle-hole polarization propagator in the Hartree scheme, at zero energy in the limit of vanishing momentum. The derivative of the baryonic density is obtained by taking the derivative with respect to $c$ of Eq. (36), with the result

$$
\left(\frac{\partial \rho}{\partial c}\right)_{\mu}=\left(g_{S}^{*}\left(\frac{\partial \bar{S}}{\partial c}\right)_{\mu}+\frac{E_{F}^{*}}{M_{N}^{*}} X^{(\pi)}\right) \Pi_{0}(0)\left(1-V_{V} \Pi_{0}(0)\right)^{-1}
$$

It follows that $\chi_{S}^{\text {nuclear }}$ can be written in the following form which displays the propagator of the scalar field and the role of the pion loops in this propagation:

$$
\begin{aligned}
\chi_{S}^{\text {nuclear }}= & -2 \frac{\langle\bar{q} q\rangle_{\mathrm{vac}}^{2}}{f_{\pi}^{2}}\left(\frac{1}{m_{\sigma}^{* 2}}-\frac{1}{m_{\sigma}^{* 2}}\right. \\
& \left.\times\left(\frac{\sigma_{N}^{(\pi)}+\sigma_{N}^{(\sigma)}}{\sigma_{N}^{(\sigma)}}\right)_{\text {eff }}^{2} \Pi_{S S}(0) \frac{1}{m_{\sigma}^{* 2}}\right) .
\end{aligned}
$$

Here $\Pi_{S S}(0)$ is the full scalar polarization propagators (in which we include the coupling constant):

$$
\Pi_{S S}(0)=g_{S}^{* 2} \frac{M_{N}^{*}}{E_{F}^{*}} \Pi_{0}(0)\left[1-V_{\text {res }} \Pi_{0}(0)\right]^{-1},
$$

where $V_{\text {res }}$ is the residual interaction:

$$
V_{\mathrm{res}}=\frac{E_{F}^{*}}{M_{N}^{*}}\left(\frac{g_{\omega}^{2}}{m_{\omega}^{2}}+\frac{\partial^{2} \varepsilon^{F C}}{\partial \rho^{2}}\right)-\frac{M_{N}^{*}}{E_{F}^{*}} \frac{g_{S}^{* 2}}{m_{\sigma}^{* 2}} .
$$

This expression differs from the mean-field one of our previous work [5] by the presence of the term in $\partial^{2} \varepsilon^{F C} / \partial \rho^{2}$. In expression (51) we have introduced quantities that we denote effective sigma commutators with the explicit expressions:

$$
\begin{aligned}
\left(\sigma_{N}\right)_{\mathrm{eff}}= & \left(\sigma_{N}^{(\pi)}\right)_{\mathrm{eff}}+\left(\sigma_{N}^{(\sigma)}\right)_{\mathrm{eff}}=\frac{M_{N}^{*}}{E_{F}^{*}} \sigma_{N}^{(\pi)}+\frac{m_{\pi}^{2}}{2} \frac{\partial\left\langle\Phi^{2}\right\rangle^{F C}}{\partial \rho} \\
& +\frac{M_{N}^{*}}{E_{F}^{*}} f_{\pi} g_{S}^{*} \frac{m_{\pi}^{2}}{m_{\sigma}^{* 2}} .
\end{aligned}
$$

It is interesting to compare it with the effective sigma commutator governing the evolution of the chiral condensate, 
which is

$$
\begin{aligned}
\left(\tilde{\sigma}_{N}\right)_{\mathrm{tot}} & =\left(\tilde{\sigma}_{N}^{(\pi)}\right)+\left(\tilde{\sigma}_{N}^{(\pi)}\right) \\
& =\frac{\rho_{S}}{\rho} \sigma_{N}^{(\pi)}+\frac{m_{\pi}^{2}}{2} \frac{\left\langle\Phi^{2}\right\rangle^{F C}}{\rho}+f_{\pi} m_{\pi}^{2} \frac{\bar{s}}{\rho} .
\end{aligned}
$$

As was shown in Ref. [7], this last quantity, corresponding to the full nuclear sigma commutator per nucleon, also governs the evolution of the pseudoscalar susceptibility according to

$$
\chi_{P S}=-2 \frac{\langle\bar{q} q\rangle_{\mathrm{vac}}^{2}}{f_{\pi}^{2} m_{\pi}^{2}}\left(1-\frac{\left(\tilde{\sigma}_{N}\right)_{\mathrm{tot}} \rho}{f_{\pi}^{2} m_{\pi}^{2}}\right) .
$$

Comparing expressions (54) and (55) we see that they are not identical. They coincide to leading order in density but the higher order terms differ. In particular $\left\langle\Phi^{2}\right\rangle^{F C} / \rho$ is replaced in Eq. (54) by $\partial\left\langle\Phi^{2}\right\rangle^{F C} / \partial \rho$. We recover in this work the result of [8], that the quantity which governs the transformation of the quark scalar density fluctuations into nucleonic ones represents an effective nucleon sigma commutator which includes pion loops. In Ref. [8] it was the free nucleon one while here interactions are incorporated and we deal with effective values. Our present result thus generalizes the first order result of [8].

Coming back to expression (51) of $\chi_{S}^{\text {nuclear }}$, we point out that the pion loops only enter in the effective sigma commutator which includes medium effects. The mass, $m_{\sigma}^{*}$, which enters the denominators is instead unaffected by the pion loops. The nuclear contribution $\chi_{S}^{\text {nuclear }}$ is indeed linked to the propagation of the chiral invariant scalar field $s$ which is not coupled to pions. The pure pion cloud contribution [Eq. (44)] is totally decoupled. This point was already discussed, within a different approach in Ref. [12].

\section{NUMERICAL RESULTS AND DISCUSSION}

\section{A. Fixing the parameters}

One important constraint to fix the parameters is the pion-nucleon sigma term which has a value, $\sigma_{N} \simeq 50 \mathrm{MeV}$. In our approach it receives two contributions: one from the pion cloud, the other from the sigma meson. We point out that if our model represents a bosonized underlying NJL model, the latter contribution represents the sigma term of constituent quarks. It is interesting to make a connection with the lattice evaluations of the evolution of the nucleon mass with the pion mass. Indeed lattice simulations of the nucleon mass as a function of the squared pion mass (equivalently the quark mass) are available in the mass region beyond $m_{\pi} \simeq 400 \mathrm{MeV}$. The derivative $\partial M_{N} / \partial m_{\pi}^{2}=\sigma_{N} / m_{\pi}^{2}$ provides the nucleon sigma commutator. In turn the derivative of $\sigma_{N}$ leads to the susceptibility. Both quantities are strongly influenced by the pion cloud which has a nonanalytic behavior in the quark mass, preventing a polynomial expansion in this quantity. However, it is possible to extrapolate lattice data using chiral models of the nucleon as discussed by Thomas and collaborators and we use their recent version Ref. [21]. In their work the pionic self-energy contribution to the nucleon mass is separated out using different cutoff forms for the pion loops (Gaussian, dipole, monopole) with an adjustable parameter $\Lambda$.
They expanded the remaining part in terms of $m_{\pi}^{2}$ as follows:

$$
M_{N}\left(m_{\pi}^{2}\right)=a_{0}+a_{2} m_{\pi}^{2}+a_{4} m_{\pi}^{4}+\Sigma_{\pi}\left(m_{\pi}, \Lambda\right) .
$$

The best fit value of the parameter $a_{4}$ which fixes the susceptibility shows little sensitivity to the shape of the form factor, with a value $a_{4} \simeq-0.5 \mathrm{GeV}^{-3}$ while $a_{2} \simeq 1.5 \mathrm{GeV}^{-1}$ (see Ref. [21]), from which we can infer the nonpionic pieces of the sigma commutator:

$$
\sigma_{N}^{\text {non-pion }}=m_{\pi}^{2} \frac{\partial M}{\partial m_{\pi}^{2}}=a_{2} m_{\pi}^{2}+2 a_{4} m_{\pi}^{4} \simeq 29 \mathrm{MeV} .
$$

This number indicates the existence of a large component $\sigma_{N}$ beside the pion one, that it is natural to attribute to the scalar field. The identification of $\sigma_{N}^{\text {non-pion }}$ with $\sigma_{N}^{(\sigma)}$ of our model fixes the sigma mass to a value $m_{\sigma}=800 \mathrm{MeV}$, close to the one $\simeq 750 \mathrm{MeV}$ that we have used in our previous article [5]. As it is the ratio $g_{s} / m_{\sigma}^{2}$ which is thus determined this value of $m_{\sigma}$ is associated with the coupling constant of the linear sigma model $g_{S}=M_{N} / f_{\pi}=10$. Lowering $g_{S}$ reduces $m_{\sigma}$. With our cutoff, $\Lambda=0.98 \mathrm{GeV}$, which yields $\sigma_{N}^{(\pi)}=21.5 \mathrm{MeV}$, the total value of the sigma term is $\sigma_{N}=50.5 \mathrm{MeV}$, a quite satisfactory result.

Although the procedure becomes more uncertain, on can also try to extract the nonpionic part of the nucleon scalar susceptibility:

$$
\chi_{N S}^{\text {non-pion }}=2 \frac{\langle\bar{q} q\rangle_{\mathrm{vac}}^{2}}{f_{\pi}^{4}} \frac{\partial}{\partial m_{\pi}^{2}}\left(\frac{\sigma_{N}^{\text {non-pion }}}{m_{\pi}^{2}}\right)=\frac{\langle\bar{q} q\rangle_{\mathrm{vac}}^{2}}{f_{\pi}^{4}} 4 a_{4} .
$$

It has to be compared with the nonpionic piece of the nucleon scalar susceptibility. This can be done taking the zero density limit of Eq. (51):

$$
\begin{aligned}
\chi_{N S}^{(\sigma)} & =-2 \frac{\langle\bar{q} q\rangle_{\mathrm{vac}}^{2}}{f_{\pi}^{2}}\left(\frac{1}{m_{\sigma}^{* 2}}-\frac{1}{m_{\sigma}^{2}}\right) \frac{1}{\rho} \\
& =-2 \frac{\langle\bar{q} q\rangle_{\mathrm{vac}}^{2}}{f_{\pi}^{2}} \frac{1}{m_{\sigma}^{4}}\left(\frac{3 g_{S}}{f_{\pi}}-\kappa_{N S}\right) .
\end{aligned}
$$

This identification allows an estimate of the crucial parameter $\kappa_{N S}$. For convenience, as in [5], we introduce the dimensionless parameter $C=\left(f_{\pi}^{2} / 2 M_{N}\right) \kappa_{N S}$ and we obtain

$$
a_{4}=-\frac{f_{\pi} g_{S}}{2 m_{\sigma}^{4}}(3-2 C) .
$$

The value $a_{4} \simeq-0.5 \mathrm{GeV}^{-3}$ leads to $C \simeq 1.25$, which indicates a strong cancellation between the two components of $a_{4}$, which makes the results sensitive to the exact values of the parameters. Therefore, due to the uncertainties of the procedure, we take this value of $C$ only as indicative.

Hence for the following discussions we fix the various parameters according to $m_{\sigma}=800 \mathrm{MeV}, \Lambda=980 \mathrm{MeV}$, $g_{N N}^{\prime}=0.7, g_{N \Delta}^{\prime}=0.3, g_{\Delta \Delta}^{\prime}=0.5, C_{\rho}=2$ and the parameter associated with the nucleonic response is allowed to vary in a broad region around $C=1.25$. The vector coupling constant $g_{\omega}$ is a totally free parameter. Finally, as in Ref. [5], we also impose that the nucleonic response vanishes at the chiral restoration $\left(\bar{s}=-f_{\pi}\right)$ :

$$
\kappa_{N S}(\bar{s})=\frac{\partial^{2} M_{N}}{\partial \bar{s}^{2}}=\kappa_{N S}\left(1+\frac{\bar{s}}{f_{\pi}}\right) .
$$




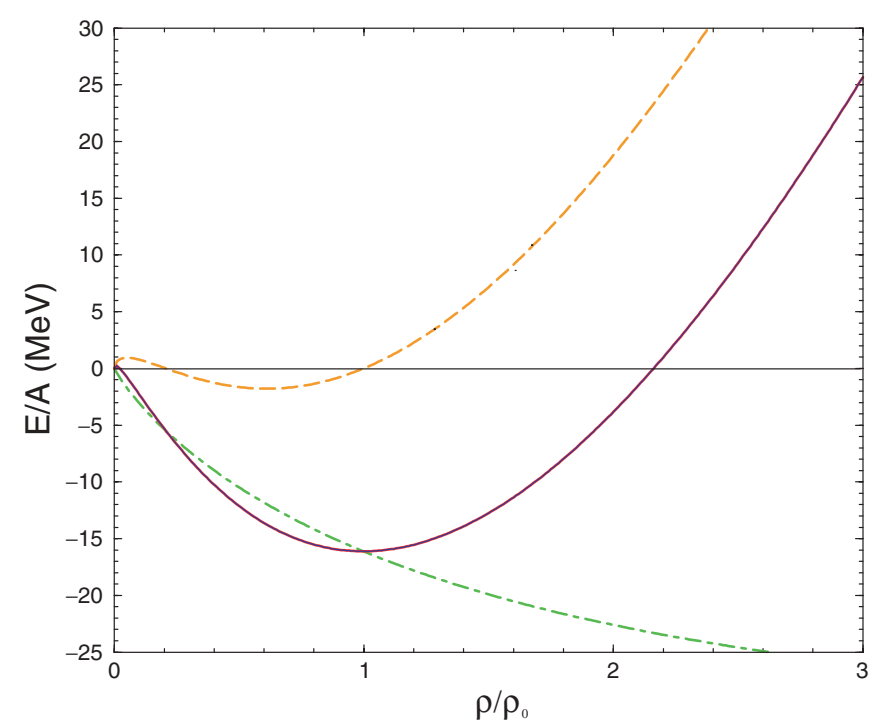

FIG. 1. (Color online) Binding energy of nuclear matter with $g_{\omega}=7.3$ and $C=0.985$ keeping only the Fock term on top of $\sigma$ and $\omega$ exchange. The full line corresponds to the full result, the dotted line represents the binding energy without the Fock term and the dot-dashed line corresponds to the contribution of the Fock term.

Accordingly, the effective nucleon mass of Eq. (3) is modified according to

$$
M_{N}^{*}(\bar{s})=M_{N}\left(1+\frac{\bar{s}}{f_{\pi}}\right)+\frac{1}{2} \kappa_{N S} \bar{s}^{2}\left(1+\frac{\bar{s}}{3 f_{\pi}}\right)
$$

and the effective scalar coupling constant becomes dependent on $\bar{s}$ :

$$
g_{S}^{*}(\bar{s})=\frac{\partial M_{N}^{*}}{\partial \bar{s}}=\frac{M_{N}}{f_{\pi}}+\kappa_{N S} \bar{s}\left(1+\frac{\bar{s}}{2 f_{\pi}}\right) .
$$

\section{B. Results with the influence of the Fock term}

The result of the calculation for the binding energy of nuclear matter in presence of the Fock term only (i.e., ignoring the correlation energy) is displayed in Fig. 1. The saturation point $\left(\rho_{0}=0.16 \mathrm{fm}^{-3}, E / A=-16.1 \mathrm{MeV}\right)$ is obtained with $C=0.985$ and $g_{\omega}=7.3$. The corresponding incompressibility is $K=254 \mathrm{MeV}$. Comparing with our previous results [5] we see that the inclusion of the Fock term somewhat improves the description since it lowers $K$. We observe in Fig. 1 that the value of the Fock term at saturation density is nearly the same as the binding energy. It turns out that, at this density, this binding comes from the transverse Fock term $\left(g^{\prime}+\right.$ rho exchange). In the longitudinal channel there is an almost exact compensation between the pion and the $g^{\prime}$ exchanges. The genuine Fock term associated with the Yukawa piece of the pion exchange represents an attractive contribution to $E / A$ of only $-4.2 \mathrm{MeV} \simeq 20 \%$ smaller than what is obtained in relativistic theories [24] where the $\pi N N$ form factor is omitted.

For completeness we also display in this subsection (Fig. 2) the nucleon and sigma masses with these values of the parameters, although they are not sensitive to the inclusion of

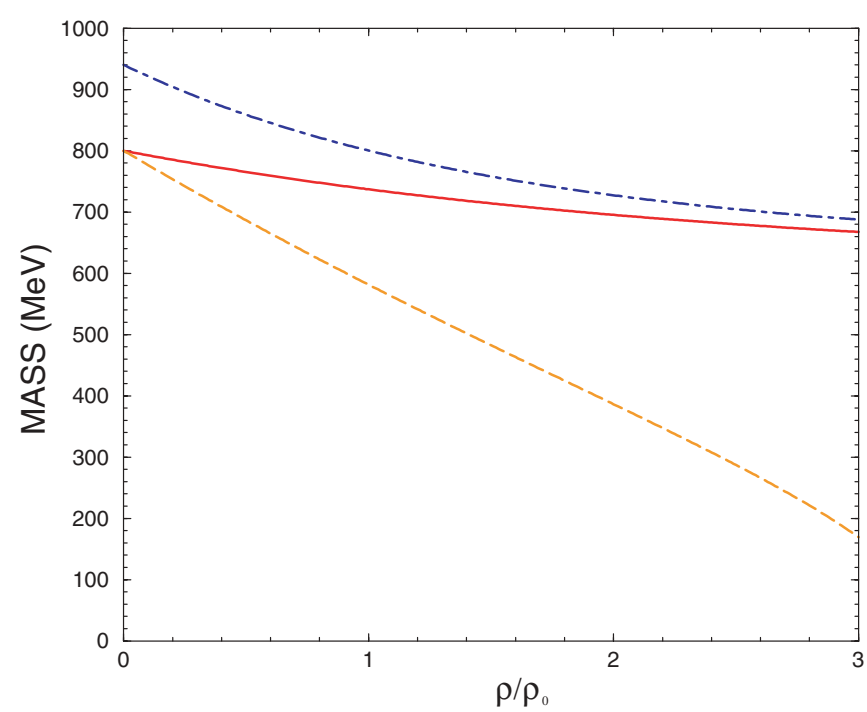

FIG. 2. (Color online) Density evolution of the nucleon (dotdashed curve) and sigma masses (full curve) with $g_{\omega}=7.3$ and $C=0.985$; the dashed curve corresponds to the sigma mass when the effect of the nucleon susceptibility is removed.

the Fock term. At normal nuclear matter density the effective nucleon mass is close to $800 \mathrm{MeV}$. As already emphasized in our previous work [5] the sigma mass remains instead quite stable, due to the introduction of the nucleonic scalar response.

The evolution of the susceptibilities is depicted in Fig. 3. The convergence effect between the pseudoscalar susceptibility (dashed line) and the scalar one (full curve) is more

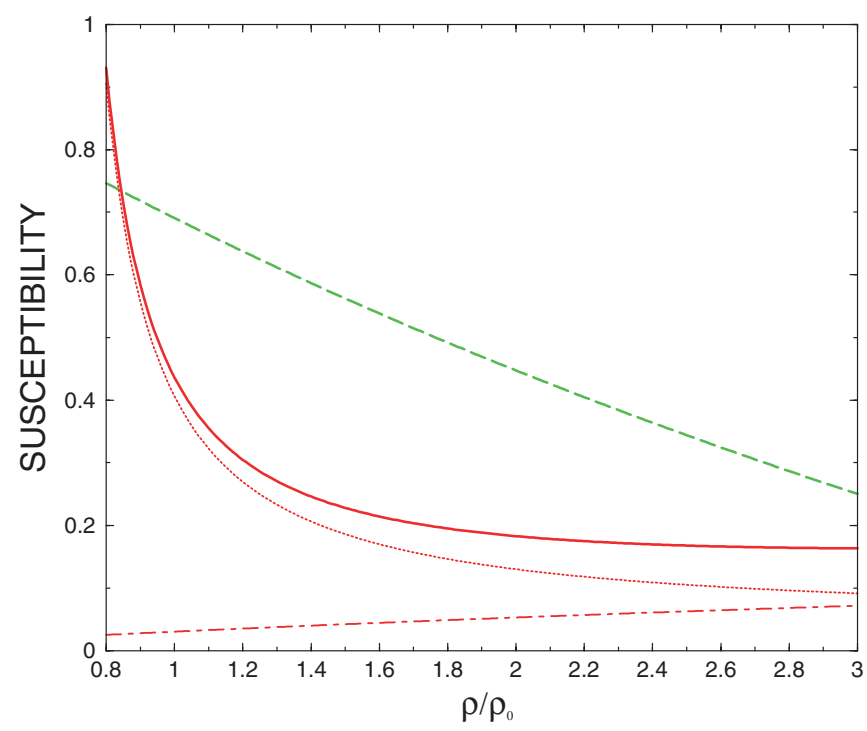

FIG. 3. (Color online) Density evolution of the QCD susceptibilities (normalized to the vacuum value of the pseudoscalar one) with $g_{\omega}=7.3$ and $C=0.985$ keeping only the Fock term on top of $\sigma$ and $\omega$ exchange. Dashed curve: pseudoscalar susceptibility. Full curve: scalar susceptibility. Dotted curve: nuclear contribution to the scalar susceptibility. Dot-dashed curve: pion loop contribution to the scalar susceptibility. 


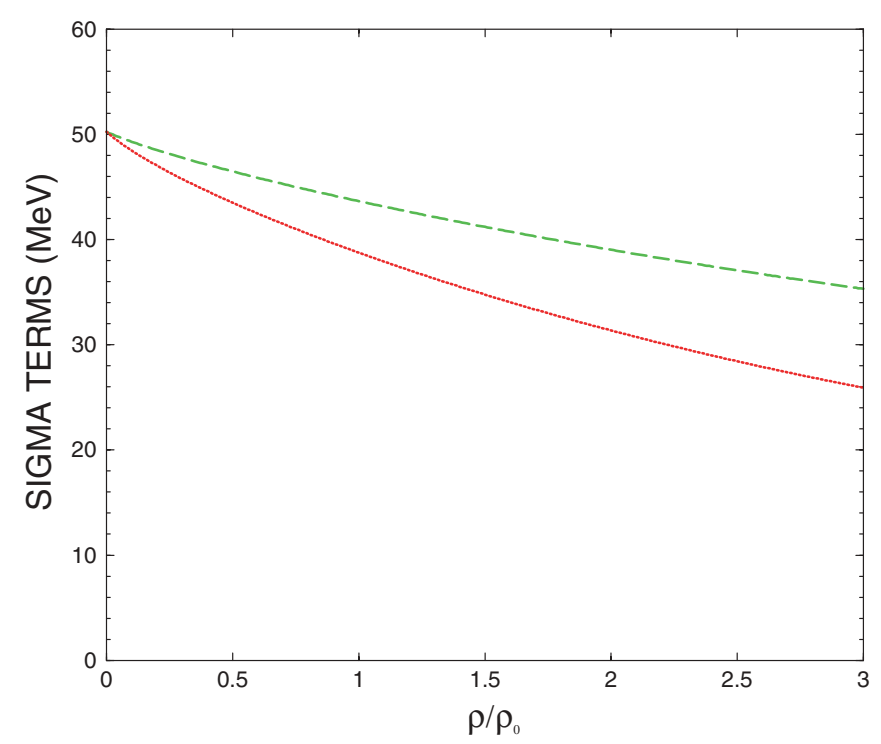

FIG. 4. (Color online) Density evolution of the full in-medium sigma term, $\left(\tilde{\sigma}_{N}\right)_{\text {tot }}$ (dotted line) and of the effective sigma term, $\left(\sigma_{N}\right)_{\text {eff }}$ (full line), with $g_{\omega}=7.3$ and $C=0.985$ keeping only the Fock term on top of $\sigma$ and $\omega$ exchange.

pronounced than in the mean field approach of our previous work [5]. The reason is clear and linked to the introduction of pion loops. Firstly the evolution of the pseudoscalar susceptibility, $\chi_{P S}$, as written in Eq. (56), is governed by the in-medium sigma term, $\left(\tilde{\sigma}_{N}\right)_{\text {tot }}$, which in the present approach receives a contribution from the pion loops which increases its value [see Eq. (55)]. Secondly, for the scalar susceptibility, the effective sigma term, $\left(\sigma_{N}\right)_{\text {eff }}$, transforming the scalar quark density fluctuations into nuclear excitations also has a pion cloud contribution [Eq. (54)] and this effect increases the nuclear part, $\chi_{S}^{\text {nuclear }}$ (dotted curve), of the scalar susceptibility. Finally the scalar susceptibility also contains a pure pion loop contribution, $\chi_{S}^{\text {pion loop }}$ (dot-dashed curve), which becomes more important at large density. For illustration we also compare in Fig. 4 the in-medium modified nucleon sigma term $\left(\tilde{\sigma}_{N}\right)_{\text {tot }}$ governing $\chi_{P S}$ (dotted curve) and the effective sigma term $\left(\sigma_{N}\right)_{\text {eff }}$ (full curve) affecting the nuclear piece $\chi_{S}^{\text {nuclear }}$.

The density behavior of the residual interaction [Eq. (53)] is displayed in Fig. 5 with separate curves for the various components (omega and sigma exchanges, Fock contribution). While the $\omega$ exchange has a very smooth density dependence, one notices the rapid variation of the sigma component associated with the dropping of the effective scalar coupling constant $g_{S}^{*}$ with increasing density. Around $\rho_{0} V_{\text {res }}$ turns from attraction into repulsion. This feature comes in part from the Fock term evolution and mostly from the behavior of the sigma exchange contribution.

\section{Influence of the correlation term}

The expression of the longitudinal contribution to the correlation energy, which is always attractive, has been given in Eq. (20). It is is depicted schematically in Fig. 6

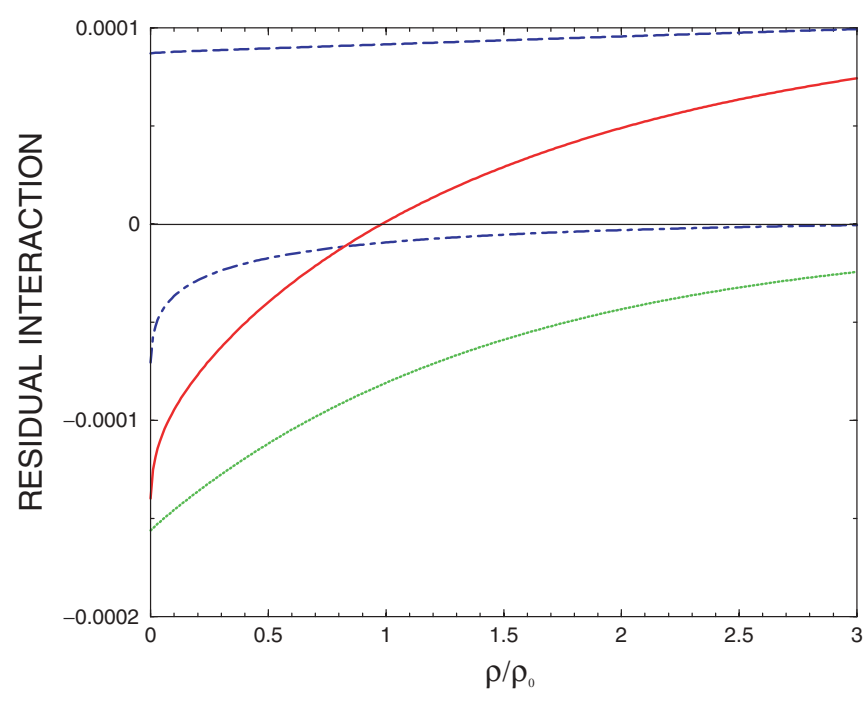

FIG. 5. (Color online) Density evolution of the residual interaction in $\mathrm{MeV}^{-2}$ (continuous line). Dashed line: omega exchange contribution. Dot-dashed line: Fock term contribution. Dotted line: sigma exchange contribution.

and involves the full longitudinal spin-isospin polarization propagator $\Pi_{L}$, which is calculated in practice within the RPA ring approximation. There is a similar expression for the transverse piece.

With the previous choice of these parameters it turns out that the numerical value of the correlation energy is close to the Fock term energy. This extra attraction has to be compensated by an additional repulsion in order to account for the saturation point. Increasing only the vector coupling constant tends to give too low a saturation density. However a slight adjustment of the sigma mass yields a correct saturation point. Taking $g_{\omega}=8$ and $m_{\sigma}=850 \mathrm{MeV}$ (corresponding to $\sigma_{N}^{\text {non-pion }}=26 \mathrm{MeV}$ ) and keeping $C=0.985$ we obtain the saturation point: $\rho_{0}=0.16 \mathrm{fm}^{-3}, E / A=-15.7 \mathrm{MeV}$. The corresponding correlation energy is $E^{\mathrm{Corr}} / A=-17.4 \mathrm{MeV}$ which divides into a longitudinal piece, $E_{L}^{\mathrm{Corr}} / A=-7.9 \mathrm{MeV}$ and a transverse one, $E_{T}^{\text {Corr }} / A=-9.5 \mathrm{MeV}$. The results of the calculation are shown in Fig. 7. We stress the importance of the transverse channel in the correlation energy as compared to the longitudinal one. The reason is the strong screening of the pion exchange by the short-range interaction in the longitudinal channel, hence a sensitivity to the $g^{\prime}$ parameters. For instance changing $g_{N \Delta}^{\prime}$ from 0.3 to 0.5 reduces further the longitudinal value to $E_{L}^{\text {Corr }} / A=-3.7 \mathrm{MeV}$ while the transverse contribution shows less sensitivity, with $E_{T}^{\text {Corr }} / A=$

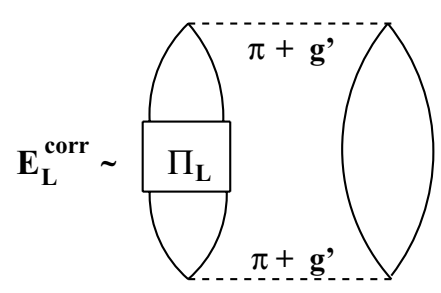

FIG. 6. Schematic representation of the longitudinal spin-isospin contribution to the correlation energy. 


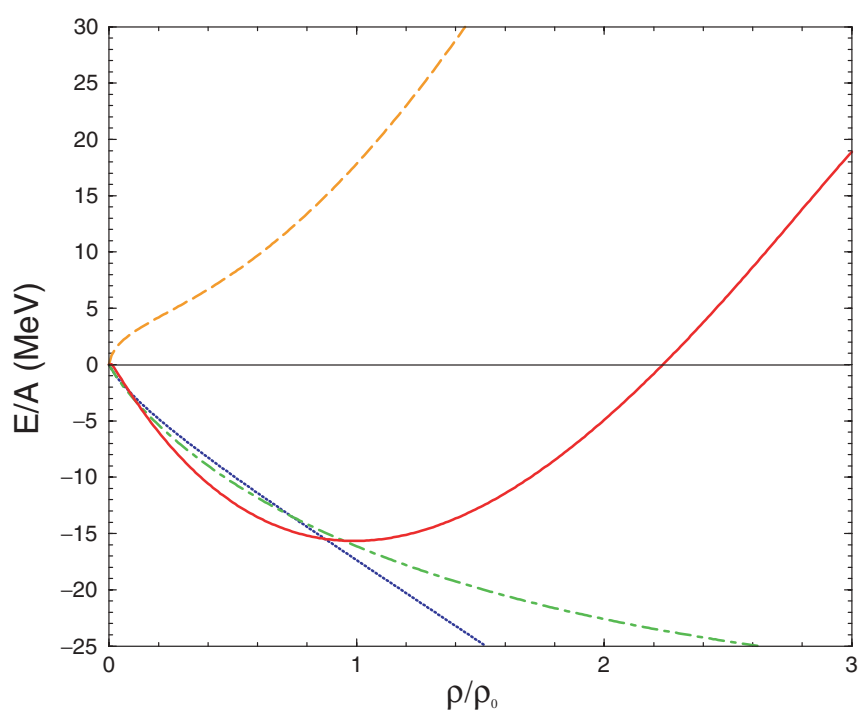

FIG. 7. (Color online) Binding energy of nuclear matter with $g_{\omega}=8, m_{\sigma}=850 \mathrm{MeV}$, and $C=0.985$ with the Fock and correlation energies on top of $\sigma$ and $\omega$ exchange. The full line corresponds to the full result, the dotted line represents the binding energy without the Fock and correlation energies and the dot-dashed line corresponds to the contribution of the Fock terms. The decreasing dotted line (always negative) represents the correlation energy.

$-7.8 \mathrm{MeV}$, such that the total value becomes $E^{\text {Corr }} / A=$ $-11.5 \mathrm{MeV}$. With the value $g_{N \Delta}^{\prime}=0.3$ the longitudinal contribution to the correlation energy is dominated by $2 \pi$ exchange with one Delta intermediate excitation. Iterated pion exchange is smaller, because of the screening effect. Therefore, as the main mechanism with one Delta is a part of the $N N$ potential, we expect the longitudinal correlation energy to be linear in density, at variance with the transverse channel where the Delta is not dominant. These behaviors are illustrated in Fig. 8. The longitudinal correlation energy has indeed a linear behavior at low densities. It deviates from linearity at high density where it becomes more attractive. This reflects the appearance of many body forces due to the increase of the nuclear pionic field, the critical opalescence effect [26] induced by the small value of $g_{N \Delta}^{\prime}$. Taking for illustration a larger $g^{\prime}$ value, $g_{N \Delta}^{\prime}=0.5$, the linearity is fulfilled in a larger density range, as shown in Fig. 9.

It is interesting to make a comparison with the chiral perturbation calculation of Ref. [9] where the effect of short-range correlation is absent. In the latter work the contribution of the iterated pion exchange is found to be $-68 \mathrm{MeV}$, the corresponding Hartree diagram coinciding with the lowest order term of the longitudinal correlation energy depicted on Fig. 6. There is nearly one order of magnitude difference between the two results, owing to the fact that in our phenomenological approach pion exchange is strongly screened by short-range correlations.

Although in detail our numerical results on the correlation energy present a sensitivity to various factors, the values of the $g^{\prime}$ parameters, the rho coupling $\left(C_{\rho}\right)$ or the form factors, especially in the transverse channel, we believe that our conclusion about the moderate importance of the correlation

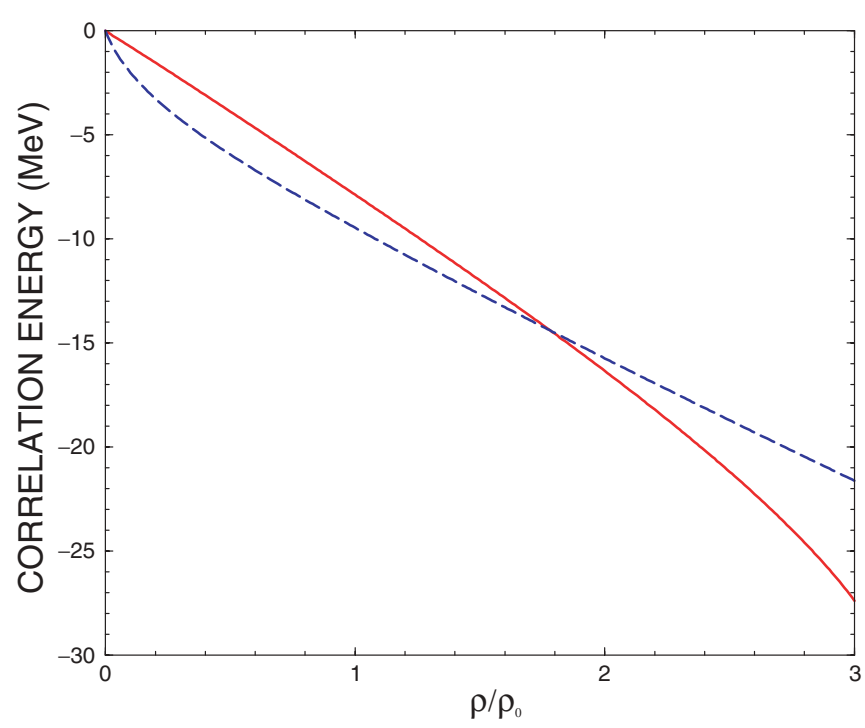

FIG. 8. (Color online) Density evolution of the longitudinal (full line) and transverse (dashed line) contributions to the correlation energy for $g_{N \Delta}^{\prime}=0.3$.

part of the energy is robust. The important point is that the rest of the interaction, as provided by lattice data, is sufficient to bind the nuclear system.

In the spirit our approach has a similarity with the density functional approach of Finelli et al. [16]. As them we have background scalar and vector fields. The first one has a connection with chiral symmetry restoration of QCD. However there is an essential difference concerning the scalar field. For Finelli et al. the full nucleon sigma commutator enters the scalar self-energy of the nucleon according to

$$
\Sigma_{S}^{(0)}=-\frac{\sigma_{N} M_{N}}{f_{\pi}^{2} m_{\pi}^{2}} \rho_{S}
$$

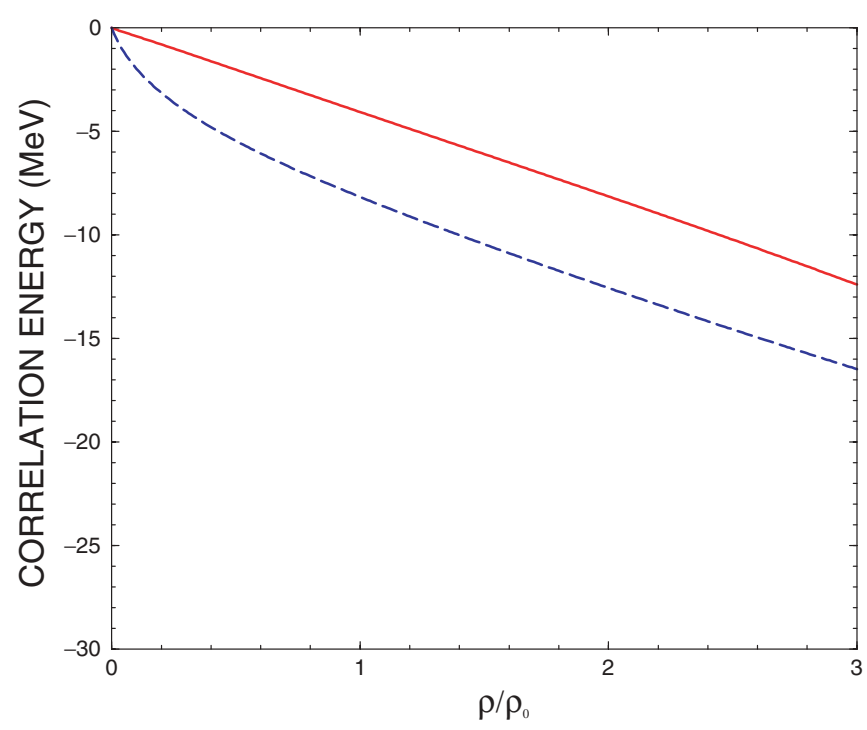

FIG. 9. (Color online) Density evolution of the longitudinal (full line) and transverse (dashed line) contributions to the correlation energy for $g_{N \Delta}^{\prime}=0.5$. 


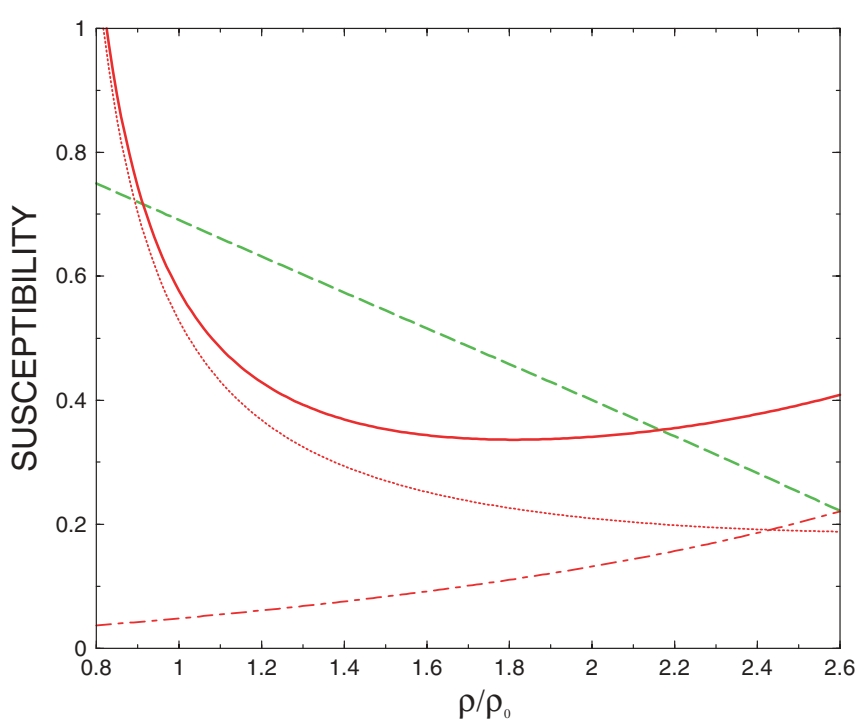

FIG. 10. (Color online) Density evolution of the QCD susceptibilities (normalized to the vacuum value of the pseudoscalar one) with $g_{\omega}=8, m_{\sigma}=850 \mathrm{MeV}$ and $C=0.985$ with the Fock and correlation terms on top of $\sigma$ and $\omega$ exchange. Dashed curve: pseudoscalar susceptibility. Full curve: scalar susceptibility. Dotted curve: nuclear contribution to the scalar susceptibility. Dot-dashed curve: pion loop contribution to the scalar susceptibility.

In our case instead, only part of the nucleon sigma commutator enters this self-energy. It is the part which originates from the chiral invariant field $s$. To leading order in density we have

$$
\Sigma_{S}^{(0)}=M_{N} \frac{\bar{s}}{f_{\pi}}=-\frac{\sigma_{N}^{(\sigma)} M_{N}}{f_{\pi}^{2} m_{\pi}^{2}} \rho_{S} .
$$

The other component from the nucleon pion cloud, $\sigma_{N}^{(\pi)} \simeq$ $21 \mathrm{MeV}$ should not enter the mass evolution as it is canceled by other terms, as imposed by chiral constraints. This point was emphasized in several works, see, e.g., Refs. [12,27]. Accordingly the link between our nuclear scalar field and QCD does not occur through the total nucleon sigma commutator but only through part of it, its nonpionic piece. Information on this last quantity can be obtained from the lattice result on the nucleon evolution with pion mass, once the pionic contribution is separated out, as done in Ref. [21] and explained in Sec. IV. Our result is therefore not totally model independent but this limitation is imposed by chiral constraints [28].

For the evaluation of the scalar susceptibility including correlation terms, we make the assumption that the pionic correlation energy density and the correlated part of the pion scalar density both go as $\rho^{2}$, a reasonable approximation in view of the results shown on Fig. 8. The net conclusion is that the convergence effect between the scalar and pseudoscalar susceptibilities is much more pronounced as shown in Fig. 10 (compared with Fig. 3). One reason is that the pionic piece of the scalar susceptibility, $\chi_{S}^{\text {pion loop }}$, is significantly increased by the RPA correlations, a conclusion already reached in our previous work [12]. Another reason comes from the fact that the full in-medium sigma term $\left(\tilde{\sigma}_{N}\right)_{\text {tot }}$, governing the pseudoscalar susceptibility and the effective sigma term, $\left(\sigma_{N}\right)_{\text {eff }}$ affecting the nuclear piece of the scalar susceptibility

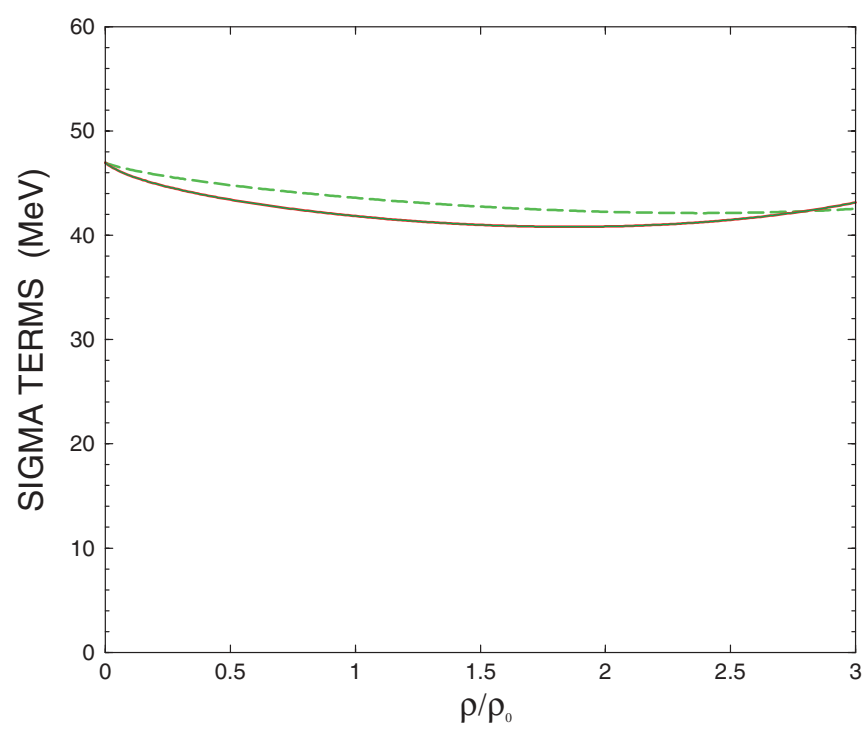

FIG. 11. (Color online) Density evolution of the full in medium sigma term, $\left(\tilde{\sigma}_{N}\right)_{\text {tot }}$ (dotted line) and of the effective sigma term, $\left(\sigma_{N}\right)_{\text {eff }}$ (full line),with $g_{\omega}=8, m_{\sigma}=850 \mathrm{MeV}$ and $C=0.985$ with the Fock and correlation terms on top of $\sigma$ and $\omega$ exchange.

remain relatively stable with the density, as depicted in Fig. 11, while they significantly decreased in Fig. 4.

\section{CONCLUSION}

We have studied in this work a relativistic nuclear model based on a chiral version of the $\sigma$ and $\omega$ exchange model. We have worked beyond the mean field approximation introducing the pion loops. Our goal is to reach a consistent description of matter which can apply at densities larger than the saturation one by introducing the pion loops on top of a mean field approach. The aim is to reach the best possible description in the framework of the model. The pion is an important actor of the nuclear dynamics and a credible theory should incorporate its effect.

In establishing the model and fixing its parameters we have kept contact with other domains. In particular we introduce beside the pion the short-range components, which are dissociable from the pion in the spin-isospin residual interaction. They are embedded in the Landau-Migdal parameters and for their values we have used the most recent information. They favor a clear deviation from universality, with a small value of $g_{N \Delta}^{\prime} \simeq 0.3$. We have also insured the compatibility of our parameters with information from QCD. One important constraint is the total nucleon sigma commutator. But more information is also available from the lattice results on the evolution of the nucleon mass with the quark mass. From the analysis of these data by Thomas et al. [21] we extracted the nonpionic piece of the sigma commutator. In our model this is the scalar field contribution and it fixes the sigma mass to be used in our inputs. Another piece of information is obtained from the higher term of the expansion, even if we take this information as indicative. Combining the information from spin-isospin physics and from QCD lattice results we can reach a tenable description of the saturation properties. 
From spin-isospin physics, we have taken the values of the Landau-Migdal $g^{\prime}$ parameters to be used in association with pion and rho exchanges. They limit the value of the correlation energy from the pion loops through a suppression of pion exchange by the short-range component. On the other hand the value of the nonpionic piece of the sigma commutator from lattice QCD, which fixes the sigma mass, provides the attraction needed for saturation to occur. Moreover the higher term $a_{4}$ of the nucleon mass expansion strongly limits the many-body effects that can occur in the propagation of the nuclear scalar field. Otherwise the tadpole terms chiral theories alone would induce too much attraction and destroy saturation. It is quite remarkable than the parameters which reproduces the saturation properties are compatible to the lattice ones. Nowhere have we faced a contradiction. It was not a priori obvious that the amount of attraction needed from the scalar field fits the value of the nonpionic sigma commutator from lattice data. Nor that the amount of cancellation necessary in the medium effects of the sigma propagation from the nucleon scalar response is reproduced in the expansion of the lattice results, with values of the parameter $C$ remarkably close ( $C=1.25$ for the lattice results versus $C \simeq 1$ in our fit). This consistency gives credibility to the description.

In more details, we have investigated the role of the Fock term and of the correlations. For the second one we have found a moderate value, $\simeq-17 \mathrm{MeV}$ at $\rho_{0}$ due to a suppression of the pion contribution by the short-range component in such a way that the transverse channel linked to rho exchange becomes dominant. The longitudinal component of the correlation energy is linear in density but it evolves faster at high densities, signalling the possible appearance of many-body forces of attractive nature. They arise from the enhancement of the nuclear pion field, the critical opalescence effect which softens the equation of state at large densities. The compatibility of the parameters of our approach with the QCD lattice data is a support for our results on the density evolution of the QCD quantities, the quark condensate and the QCD susceptibilities. We have insured that these results are in addition fully compatible with the saturation properties of nuclear matter. For the quark condensate we find small deviations from a linear behavior with density, in spite of the inclusion of various types of many-body effects. This also applies for the pseudoscalar susceptibility which follows the condensate. The scalar susceptibility is instead very sensitive to the interactions and shows a large enhancement as compared to the free value. It even surpasses the pseudoscalar one beyond $\rho \simeq 2.2 \rho_{0}$ while in the vacuum it is much smaller.

Our description could be improved in various directions such as the incorporation of all the Fock terms (sigma and omega exchange, time component of the rho meson exchange) and a covariant description of the short-range interaction in a fully covariant framework. It would also be interesting to extend the approach to the case of asymmetric nuclear matter and neutron matter.

\section{ACKNOWLEDGMENT}

We thank P. Guichon for fruitful discussions.
[1] B. D. Serot and J. D. Walecka, Adv. Nucl. Phys. 16, 1 (1986); Int. J. Mod. Phys. E 6, 515 (1997).

[2] G. Chanfray, M. Ericson, and P. A. M. Guichon, Phys. Rev. C 63, 055202 (2001).

[3] A. K. Kerman and L. D. Miller, "Second High Energy Heavy Ion Summer Study,” LBL-3675, 1974.

[4] W. Bentz and A. W. Thomas, Nucl. Phys. A696, 118 (2001).

[5] G. Chanfray and M. Ericson, Eur. Phys. J. A 25, 151 (2005).

[6] P. A. M. Guichon, Phys. Lett. B200, 235 (1988).

[7] G. Chanfray and M. Ericson, Eur. Phys. J. A 16, 291 (2003).

[8] G. Chanfray, M. Ericson, and P. A. M. Guichon, Phys. Rev. C 68, 035209 (2003).

[9] N. Kaiser, S. Fritsch, and W. Weise, Nucl. Phys. A697, 82 (2002).

[10] M. Ichimura, H. Sakai, and T. Wakasa, Prog. Part. Nucl. Phys. 56, 446 (2006).

[11] G. Chanfray, P. Schuck, and W. Norenberg, in Proceedings of the International Workshop on Gross Properties of Nuclei and Nuclear Excitations, Hirshegg, Austria, January 1990, edited by H. Feldmaier, p. 286; Z. Aouissat, G. Chanfray, P. Schuck, and W. Norenberg, Proceedings of Corinne 90 International Workshop on Particle and Interferometry in Nuclear Collisions, Nantes, France, June, 1990 (World Scientific, Singapore).

[12] G. Chanfray, D. Davesne, M. Ericson, and M. Martini, Eur. Phys. J. A 27, 191 (2006).
[13] F. Bonnuti et al., Nucl. Phys. A677, 213 (2000); Phys. Rev. Lett. 77, 603 (1996); Phys. Rev. C 60, 018201 (1999).

[14] A. Starostin et al., Phys. Rev. Lett. 85, 5539 (2000).

[15] J. G. Messchendorp et al., Phys. Rev. Lett. 89, 222302 (2002).

[16] P. Finelli, N. Kaiser, D. Vretenar, and W. Weise, Nucl. Phys. A770, 1 (2006).

[17] S. Lawley, W. Bentz, and A. W. Thomas, J. Phys. G 32, 667 (2006).

[18] G. Chanfray, M. Ericson, and M. Oertel, Phys. Lett. B563, 61 (2003).

[19] J. Delorme, M. Ericson, and T. E. O. Ericson, Phys. Lett. B291, 379 (1992).

[20] G. Hohler and E. Pietarinen, Nucl. Phys. B95, 210 (1975).

[21] A. W. Thomas, P. A. M. Guichon, D. B. Leinweber, and R. D. Young, Prog. Theor. Phys. Suppl. 156, 124 (2004).

[22] I. Jameson, A. W. Thomas, and G. Chanfray, J. Phys. G 18, L159 (1992).

[23] M. C. Birse and J. E. McGovern, Phys. Lett. B292, 242 (1992).

[24] A. Bouissy, J. F. Mathiot, Nguyen Van Giai, and S. Marcos, Phys. Rev. C 36, 380 (1987).

[25] G. Chanfray and D. Davesne, Nucl. Phys. A646,125 (1999).

[26] J. Delorme and M. Ericson, Phys. Lett. B76, 182 (1978).

[27] M. Birse, Phys. Rev. C 49, 2212 (1996).

[28] M. Birse, Phys. Rev. C 53, 2048 (1996); Acta Phys. Pol. B 29, 2357 (1998). 